PAPER • OPEN ACCESS

\title{
An inverse potential problem for subdiffusion: stability and reconstruction ${ }^{*}$
}

To cite this article: Bangti Jin and Zhi Zhou 2021 Inverse Problems 37015006

View the article online for updates and enhancements.

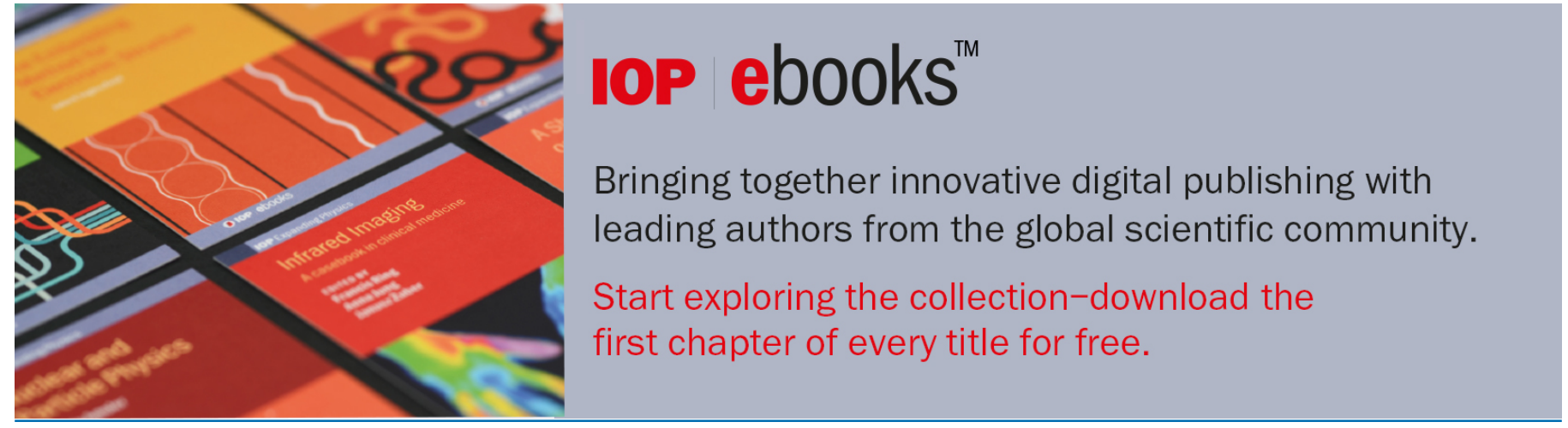

This content was downloaded from IP address 193.60 .238 .99 on 15/01/2021 at 06:23 


\title{
An inverse potential problem for subdiffusion: stability and reconstruction*
}

\author{
Bangti $\operatorname{Jin}^{1, * *}$ (1) and Zhi Zhou ${ }^{2}$ (i) \\ ${ }^{1}$ Department of Computer Science, University College London, Gower Street, \\ London WC1E 6BT, United Kingdom \\ 2 Department of Applied Mathematics, The Hong Kong Polytechnic University, \\ Kowloon, Hong Kong Special Administrative Region of China \\ E-mail: b.jin@ucl.ac.uk, bangti.jin@gmail.com and zhizhou@ polyu.edu.hk
}

Received 1 June 2020, revised 7 September 2020

Accepted for publication 8 September 2020

Published 3 December 2020

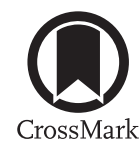

\begin{abstract}
In this work, we study the inverse problem of recovering a potential coefficient in the subdiffusion model, which involves a Djrbashian-Caputo derivative of order $\alpha \in(0,1)$ in time, from the terminal data. We prove that the inverse problem is locally Lipschitz for small terminal time, under certain conditions on the initial data. This result extends the result in [6] for the standard parabolic case to the fractional case. The analysis relies on refined properties of two-parameter Mittag-Leffler functions, e.g., complete monotonicity and asymptotics. Further, we develop an efficient and easy-toimplement algorithm for numerically recovering the coefficient based on (preconditioned) fixed point iteration and Anderson acceleration. The efficiency and accuracy of the algorithm is illustrated with several numerical examples.
\end{abstract}

Keywords: inverse potential problem, subdiffusion, stability, numerical reconstruction

(Some figures may appear in colour only in the online journal)

\footnotetext{
${ }^{*}$ The work of B Jin is supported by UK EPSRC Grant EP/T000864/1, and the research of Z Zhou is supported by Hong Kong RGC Grant (No. 15304420).

${ }^{* *}$ Author to whom any correspondence should be addressed.
}

Original content from this work may be used under the terms of the Creative Commons Attribution 4.0 licence. Any further distribution of this work must maintain attribution to the author(s) and the title of the work, journal citation and DOI. 


\section{Introduction}

Let $\Omega \subset \mathbb{R}^{d}(d=1,2,3)$ be a smooth open bounded domain with a boundary $\partial \Omega$. Consider the following initial boundary value problem for subdiffusion:

$$
\left\{\begin{aligned}
\partial_{t}^{\alpha} u & =\Delta u+q(x) u, & & \text { in } \Omega \times(0, T], \\
u(\cdot, 0) & =u_{0}, & & \text { in } \Omega, \\
u & =0, & & \text { on } \partial \Omega \times(0, T],
\end{aligned}\right.
$$

where $T>0$ is the final time and $u_{0}$ is the initial data. The notation $\partial_{t}^{\alpha} u$ denotes the Djrbashian-Caputo derivative of order $\alpha \in(0,1)$ (in time), defined by [22, p. 91]

$$
\partial_{t}^{\alpha} u(t)=\frac{1}{\Gamma(1-\alpha)} \int_{0}^{t}(t-s)^{-\alpha} u^{\prime}(s) \mathrm{d} s,
$$

where

$$
\Gamma(z)=\int_{0}^{\infty} s^{z-1} \mathrm{e}^{-s} \mathrm{~d} s, \quad \text { for } \mathfrak{R} z>0
$$

denotes Euler's Gamma function. For smooth functions $u$, the fractional derivative $\partial_{t}^{\alpha} u$ recovers the usual first-order derivative $u^{\prime}(t)$ as $\alpha \rightarrow 1^{-}$. The function $q$ refers to the radiativity or reaction coefficient or potential in the standard parabolic case, dependent of the specific applications. Throughout, we denote by $u(q)$ the solution of problem (1.1) that corresponds to a given potential $q \in L^{2}(\Omega)$.

The model (1.1) is a direct extension of the standard subdiffusion model, which has a trivial potential $q$ (i.e., $q \equiv 0$ ), and can faithfully describe anomalously slow diffusion processes. At a microscopical level, standard subdiffusion can be described by continuous time random walk, where the waiting time distribution between consecutive jumps is heavy tailed with a divergent mean, in a manner similar to Brownian motion for normal diffusion, and the governing equation for the probability density function of the particle appearing at certain time instance $t$ and space location $x$ is of the form. Subdiffusion has been observed in several applications in engineering, physics and biology, e.g., thermal diffusion in fractal domains [31], and dispersive ion transport in column experiments [11]; see the review [29] for physical motivation and an extensive list of physical applications; see also the works $[12,41]$ for the derivation of reaction-subdiffusion models within the framework of continuous time random walk.

In this work, we study the following inverse problem for the model (1.1): given a function $g$, recover $q \in L^{2}(\Omega)$ such that

$$
u(q)(\cdot, T)=g \quad \text { in } \Omega .
$$

The direct problem for $q \in L^{2}(\Omega)$ has not been extensively studied, and we give a study in section 2 via an operator theoretic formulation. Let $A=-\Delta$, with its domain $D(A)=H_{0}^{1}(\Omega) \cap H^{2}(\Omega)$, and the graph norm denoted by $\|\cdot\|_{D(A)}$. If $\omega$ is an open subset of the domain $\Omega$, we identify

$$
L^{2}(\omega)=\left\{f \in L^{2}(\Omega):\left(1-\chi_{\omega}\right) f=0\right\}
$$

where $\chi_{\omega}$ denotes the characteristic function of the subset $\omega$. We denote by $\lambda_{1}$ the smallest eigenvalue of $A$, and $\bar{\varphi}_{1}$ the corresponding nonnegative eigenfunction, normalized by 
$\left\|\bar{\varphi}_{1}\right\|_{L^{\infty}(\Omega)}=1$. Further, let

$$
c_{\alpha}=\sup _{t \geqslant 0} t E_{\alpha, \alpha}(-t)
$$

where $E_{\alpha, \alpha}(z)$ is the two-parameter Mittag-Leffler function defined in (2.2) below. This constant plays a crucial role in the analysis. Proposition 3.1 gives an upper bound on $c_{\alpha}$, which implies $c_{\alpha}<\alpha$.

Then the following stability estimate holds: for small time $T$, the inverse problem is locally Lipschitz stable. The proof of the theorem employs the implicit function theorem, and certain estimates on the solution operators with sharp constants, which in turn uses heavily refined properties of Mittag-Leffler functions; see section 3 for the detailed proof.

Theorem 1.1. Let $\frac{3}{4}<\gamma<1,0<\epsilon<1-\frac{c_{\alpha}}{\alpha}, \mu_{0}, \mu_{1}>0$ such that $1 \leqslant \frac{\mu_{1}}{\mu_{0}}<\frac{(1-\epsilon) \alpha}{c_{\alpha}}$. Let $u_{0} \in D\left(A^{1+\gamma}\right)$, with

$$
\mu_{0} \lambda_{1} \bar{\varphi}_{1} \leqslant-\Delta u_{0} \leqslant \mu_{1} \lambda_{1} \bar{\varphi}_{1}
$$

and set $\omega=\left\{x \in \Omega: \bar{\varphi}_{1}(x) \geqslant 1-\epsilon\right\}$. Then there exists a constant $\theta>0$ depending only on $\frac{\mu_{1}}{(1-\epsilon) \mu_{0}}$ and $\alpha$ such that if $\lambda_{1} T^{\alpha}<\theta$, then there is $V$, a neighborhood of $O$ in $L^{2}(\omega)$ and a constant $c$ such that

$$
\left\|q_{1}-q_{2}\right\|_{L^{2}(\omega)} \leqslant c\left\|u\left(q_{1}\right)(T)-u\left(q_{2}\right)(T)\right\|_{D(A)}, \quad \forall q_{1}, q_{2} \in V .
$$

Remark 1.1. The regularity condition $u_{0} \in D\left(A^{1+\gamma}\right)$ is to ensure the well-posedness of the direct problem with $q \in L^{2}(\Omega)$. The condition (1.4) is to ensure pointwise lower and upper bounds on the solution $u(0)(T)$, and the set of $u_{0}$ satisfying (1.4) is a convex subset of $D\left(A^{1+\gamma}\right)$. The condition $\lambda_{1} T^{\alpha}<\theta$ dictates that either $T$ or $\lambda_{1}$ should be sufficiently small, the latter of which holds if the domain $\Omega$ is large, since $\lambda_{1}$ tends to zero as the volume of $\Omega$ tends to infinity [8].

We also develop a simple algorithm to numerically recover the potential $q$. It is based on preconditioned fixed point iteration given in (4.1), and employs Anderson acceleration [2] to speed up the convergence. It extends an existing scheme proposed in [34] for the standard parabolic problem to subdiffusion, but enhanced by the preconditioner $A^{-1}$ for better numerical stability and acceleration via Anderson acceleration. The algorithm is straightforward to implement, since it involves solving one direct problem at each iteration, and generally applicable (no sign restriction, no condition on the initial data), and when equipped with the discrepancy principle $[9,14]$, it is also accurate for both subdiffusion and normal diffusion. We provide several numerical experiments to confirm the efficiency and accuracy of the algorithm, and to illustrate the behavior of the inverse problem. The stability result in theorem 1.1 and the reconstruction algorithm represent the main contributions of this work.

Now we discuss several existing works. Inverse problems for subdiffusion are of relative recent nature, initiated by the pioneering work [4] for recovering the diffusion coefficient from lateral Cauchy data (in the one-dimensional case) using Sturm-Liouville theory; see the work [19] for an overview. The inverse potential problem for the model (1.1) has also been analyzed in several works [18, 20, 21, 30, 42]. Miller and Yamamoto [30] proved the unique recovery from data on a space-time subdomain, using an integral transformation. Zhang and Zhou [42] discussed the unique recovery using a fixed point argument [13], and derived error estimates in the presence of data noise. Kaltenbacher and Rundell [20] gave the well-posedness of the 
direct problem and also proved the invertibility of the linearized map from the space $L^{2}(\Omega)$ to $H^{2}(\Omega)$ under the condition $u_{0}>0$ in $\Omega$ and $q \in L^{\infty}(\Omega)$ using a Paley-Wiener type result, where the condition $q \in L^{\infty}(\Omega)$ plays a central in the proof, which invokes a type of strong maximum principle. Further, they developed (frozen) Newton and Halley type iterative schemes for numerically recovering the coefficient $q$ from the terminal data, and proved their convergence. Kian and Yamamoto [21] derived a stability result for recovering a space-time dependent potential coefficient from lateral Cauchy data. It is worth noting that the parabolic counterpart of the inverse problem (1.2) has been extensively studied [5, 6, 13, 23, 33]. Isakov [13] proved the existence and uniqueness for the inverse problem using strong maximum principle, and proposed a constructive algorithm based on fixed point iteration. Choulli and Yamamoto [5] proved a generic well-posedness result in a Holderian space, by introducing a scalar parameter in the leading elliptic term $\Delta u$. Later, they [6] analyzed the inverse problem in a Hilbert space setting. Theorem 1.1 represents an extension of the result in [6] to the subdiffusion case. Note that due to the drastic difference in solution operators, i.e., the fractional case involves Mittag-Leffler functions, the extension is nontrivial. We refer interested readers to [25, 26, 36] and references therein for related inverse source problems, which are often employed to analyze the generic well-posedness for the inverse potential problem.

The rest of the paper is organized as follows. In section 2, we discuss the well-posedness of the direct problem, and prove that for every $q \in L^{2}(\Omega)$, there exists a unique classical solution, for suitably smooth initial data $u_{0}$. Then in section 3 , we give the proof of theorem 1.1 . Next, we develop the fixed point algorithm and present its preliminary properties in section 4 . Last, we provide several numerical experiments to illustrate feasibility of the reconstruction algorithm. Throughout, $(\cdot, \cdot)$ denotes the $L^{2}(\Omega)$ inner product, and $H^{s}(\Omega)$ denotes the usual Sobolev space [1]. The notation $c$ denotes a generic constant which may change at each occurrence, but it is always independent of the coefficient $q$.

\section{Well-posedness of the Cauchy problem}

First we study the well-posedness of the following abstract Cauchy problem:

$$
\left\{\begin{aligned}
\partial_{t}^{\alpha} u(t)+A u(t) & =q u(t), \quad \text { in }(0, T), \\
u(0) & =u_{0}
\end{aligned}\right.
$$

It is a reformulation of the direct problem (1.1) into an operator form. We prove that for suitably smooth $u_{0}$ and any $q \in L^{2}(\Omega)$, problem (2.1) has a unique classical solution $u=u(q) \in$ $C^{\alpha}\left([0, T] ; L^{2}(\Omega)\right) \cap C([0, T] ; D(A))$. The analysis is based on a 'perturbation' argument, developed recently in [17] for the numerical analysis of nonlinear subdiffusion problems, where Banach fixed point theorem and the argument of equivalent norm family play an important role (see, e.g., [7, chapter 3]); see also [20] for a well-posedness result under slightly different assumptions on the potential $q$.

Specifically, let $\left\{\left(\lambda_{j}, \varphi_{j}\right)\right\}_{j=1}^{\infty}$ be the eigenpairs of the operator $A$, with the eigenvalues $\lambda_{j}$ ordered nondecreasingly and multiplicity counted, and $\left\{\varphi_{j}\right\}_{j=1}^{\infty}$ form an orthonormal basis of $L^{2}(\Omega)$. For any $\gamma>0$, the notation $D\left(A^{\gamma}\right)$ denotes the domain of the fractional power $A^{\gamma}$, with the graph norm $\|\cdot\|_{D\left(A^{\gamma}\right)}$, given by

$$
\|v\|_{D(A \gamma)}=\left(\sum_{j=1}^{\infty} \lambda_{j}^{2 \gamma}\left(v, \varphi_{j}\right)^{2}\right)^{\frac{1}{2}} .
$$


By viewing $q u(t)$ as the inhomogeneous term and applying Duhamel's principle, we deduce that the solution $u(t)$ satisfies

$$
u(t)=U(t)+\int_{0}^{t} E(t-s) q u(s) \mathrm{d} s,
$$

where $U(t)=F(t) u_{0}$, and the solution operators $F(t)$ and $E(t)$ are defined by [17]

$$
\begin{aligned}
& F(t) v=\sum_{j=1}^{\infty} E_{\alpha, 1}\left(-\lambda_{j} t^{\alpha}\right)\left(\varphi_{j}, v\right) \varphi_{j}, \\
& E(t) v=\sum_{j=1}^{\infty} t^{\alpha-1} E_{\alpha, \alpha}\left(-\lambda_{j} t^{\alpha}\right)\left(\varphi_{j}, v\right) \varphi_{j} .
\end{aligned}
$$

Here $E_{\alpha, \beta}(z)$ refers to the two-parameter Mittag-Leffler function, defined by [22]

$$
E_{\alpha, \beta}(z)=\sum_{k=0}^{\infty} \frac{z^{k}}{\Gamma(k \alpha+\beta)}, \quad z \in \mathbb{C} .
$$

The next lemma collects smoothing properties of the operators $F$ and $E$. The notation $\|\cdot\|$ denotes the operator norm on $L^{2}(\Omega)$.

Lemma 2.1. For the operators $F(t)$ and $E(t)$, the following estimates hold

$$
\begin{gathered}
\|F(t)\| \leqslant E_{\alpha, 1}\left(-\lambda_{1} t^{\alpha}\right), \quad\|E(t)\| \leqslant t^{\alpha-1} E_{\alpha, \alpha}\left(-\lambda_{1} t^{\alpha}\right), \\
\left\|A^{\theta} E(t)\right\| \leqslant c_{\alpha, \theta} t^{(1-\theta) \alpha-1} .
\end{gathered}
$$

where the constant $c_{\alpha, \theta}>0$ depends on $\alpha$ and $\theta$.

Proof. The first estimate follows from the fact that $E_{\alpha, 1}(-t)$ is completely monotone [32]:

$$
\begin{aligned}
\|F(t) v\|_{L^{2}(\Omega)}^{2} & =\sum_{j=1}^{\infty} E_{\alpha, 1}\left(-\lambda_{j} t^{\alpha}\right)^{2}\left(\varphi_{j}, v\right)^{2} \\
& \leqslant E_{\alpha, 1}\left(-\lambda_{1} t^{\alpha}\right)^{2} \sum_{j=1}^{\infty}\left(\varphi_{j}, v\right)^{2}=E_{\alpha, 1}\left(-\lambda_{j} t^{\alpha}\right)^{2}\|v\|_{L^{2}(\Omega)}^{2} .
\end{aligned}
$$

The second follows similarly since $E_{\alpha, \alpha}(-t)$ is also completely monotone, and the last is known from [17, lemma 3.4].

Now we can specify the function analytic setting. Let $0<\beta<(1-\gamma) \alpha$ be fixed and set

$$
X=C^{\beta}\left([0, T] ; D\left(A^{\gamma}\right)\right) \cap C([0, T] ; D(A)),
$$

with the norm given by

$$
\|v\|_{X}=\|v\|_{C^{\beta}\left([0, T] ; D\left(A^{\gamma}\right)\right)}+\|v\|_{C([0, T] ; D(A))} .
$$

Then for every $q \in L^{2}(\Omega)$, we define an associated operator $L(q)$ by

$$
[L(q)] f(t)=\int_{0}^{t} E(t-s) q f(s) \mathrm{d} s, \quad \forall f \in C^{\beta}\left([0, T] ; D\left(A^{\gamma}\right)\right) .
$$


The next result gives the mapping property of the operator $L(q)$.

Lemma 2.2. For any $q \in L^{2}(\Omega), L(q)$ maps $C^{\beta}\left([0, T] ; D\left(A^{\gamma}\right)\right)$, with $\gamma>\frac{3}{4}$, into $X$.

Proof. Let $f \in C^{\beta}\left([0, T] ; D\left(A^{\gamma}\right)\right), q \in L^{2}(\Omega)$ and let $g(t)=q f(t), 0 \leqslant t \leqslant T$. We split the function $L(q) f$ into two terms $L(q) f=v_{1}+v_{2}$, with

$$
v_{1}(t)=\int_{0}^{t} E(t-s)(g(s)-g(t)) \mathrm{d} s \quad \text { and } \quad v_{2}(t)=\int_{0}^{t} E(t-s) g(t) \mathrm{d} s .
$$

Since $f \in C^{\beta}\left([0, T] ; D\left(A^{\gamma}\right)\right)$, by Sobolev embedding theorem $[1], g \in C^{\beta}\left([0, T] ; L^{2}(\Omega)\right)$, and thus by [35, lemma 3.4], $v_{1} \in C^{\beta}([0, T] ; D(A)) \subset X$. Next, for $t \in[0, T], \tau>0$ such that $t+\tau \leqslant T$, we have

$$
v_{2}(t+\tau)-v_{2}(t)=\int_{t}^{t+\tau} E(s) g(t+\tau) \mathrm{d} s+\int_{0}^{t} E(s)[g(t+\tau)-g(t)] \mathrm{d} s .
$$

Thus by the smoothing property of $E$ in lemma 2.1, we deduce

$$
\begin{aligned}
\left\|A^{\gamma}\left(v_{2}(t+\tau)-v_{2}(t)\right)\right\|_{L^{2}(\Omega)} \leqslant & c_{\alpha, \gamma}\|g\|_{C\left([0, T] ; L^{2}(\Omega)\right)} \int_{t}^{t+\tau} s^{(1-\gamma) \alpha-1} \mathrm{~d} s \\
& +c_{\alpha, \gamma} \tau^{\beta}\|g\|_{C^{\beta}\left([0, T] ; L^{2}(\Omega)\right)} \int_{0}^{t} s^{(1-\gamma) \alpha-1} \mathrm{~d} s .
\end{aligned}
$$

Since

$$
\int_{t}^{t+\tau} s^{(1-\gamma) \alpha-1} \mathrm{~d} s \leqslant \int_{0}^{\tau} s^{(1-\gamma) \alpha-1} \mathrm{~d} s=\frac{\tau^{(1-\gamma) \alpha}}{(1-\gamma) \alpha}
$$

we obtain

$$
\begin{aligned}
& \tau^{-\beta}\left\|A^{\gamma}\left[v_{2}(t+\tau)-v_{2}(t)\right]\right\|_{L^{2}(\Omega)} \\
& \quad \leqslant \frac{c_{\alpha, \gamma}}{(1-\gamma) \alpha}\left(\tau^{(1-\gamma) \alpha-\beta}+T^{(1-\gamma) \alpha}\right)\|g\|_{C^{\beta}\left([0, T] ; L^{2}(\Omega)\right)}
\end{aligned}
$$

Since $\beta<(1-\gamma) \alpha, v_{2} \in C^{\beta}\left([0, T] ; L^{2}(\Omega)\right)$. It remains to show $A v_{2} \in C\left([0, T] ; L^{2}(\Omega)\right)$. This follows from the identity

$$
-A v_{2}(t)=-\int_{0}^{t} A E(t-s) g(t) \mathrm{d} s=(F(t)-I) g(t),
$$

in view of the identity $\frac{\mathrm{d}}{\mathrm{d} t}(I-F(t))=A E(t)$ [17]. Since $F(t)-I$ is continuous on $L^{2}(\Omega)$, the desired assertion follows. This completes the proof of the lemma.

Lemma 2.3. If $q \in L^{2}(\Omega)$, then $I-L(q)$ has a bounded inverse in $B(X)$.

Proof. The proof proceeds by the argument of equivalent norm family (see, e.g., [7, chapter 3 , section 3.8]). Specifically, we equip the space $X$ with an equivalent family of norms $\|\cdot\|_{\lambda}$, $\lambda \geqslant 0$, defined by

$$
\begin{aligned}
\|f\|_{\lambda}= & \sup _{t \in[0, T]} \mathrm{e}^{-\lambda t}\left[\|f(t)\|_{L^{2}(\Omega)}+\left\|A^{\gamma} f(t)\right\|_{L^{2}(\Omega)}\right] \\
& +\sup _{0 \leqslant s<t \leqslant T} \mathrm{e}^{-\lambda(t+1)} \frac{\|f(s)-f(t)\|_{D\left(A^{\gamma}\right)}}{|t-s|^{\beta}}+\sup _{t \in[0, T]} \mathrm{e}^{-\lambda(t+2)}\|A f(t)\|_{L^{2}(\Omega)},
\end{aligned}
$$


which is equivalent to the norm on $X$, and then prove the invertibility by choosing $\lambda$ suitably. For $f \in X$, let $v=L(q) f$. Then by Sobolev embedding [1] and lemma 2.1,

$$
\begin{aligned}
\mathrm{e}^{-\lambda t}\|v(t)\|_{L^{2}(\Omega)} & =\mathrm{e}^{-\lambda t}\left\|\int_{0}^{t} E(t-s) q f(s) \mathrm{d} s\right\|_{L^{2}(\Omega)} \\
& \leqslant c \int_{0}^{t} \mathrm{e}^{-\lambda(t-s)}\|E(t-s)\|\|q\|_{L^{2}(\Omega)} \mathrm{e}^{-\lambda s}\|f(s)\| \|_{C\left([0, T] ; D\left(A^{\gamma}\right)\right)} \mathrm{d} s \\
& \leqslant c \int_{0}^{t} \mathrm{e}^{-\lambda s} s^{\alpha-1} \mathrm{~d} s\|q\|_{L^{2}(\Omega)}\|f\|_{\lambda} \leqslant c \lambda^{-\alpha}\|q\|_{L^{2}(\Omega)}\|f\|_{\lambda},
\end{aligned}
$$

where the last inequality follows from changing variables $\zeta=\lambda s$ by

$$
\int_{0}^{t} \mathrm{e}^{-\lambda s} s^{\alpha-1} \mathrm{~d} s=\lambda^{-\alpha} \int_{0}^{\lambda t} \mathrm{e}^{-\zeta} \zeta^{\alpha-1} \mathrm{~d} \zeta \leqslant \lambda^{-\alpha} \int_{0}^{\infty} \zeta^{\alpha-1} \mathrm{e}^{-\zeta} \mathrm{d} \zeta=\lambda^{-\alpha} \Gamma(\alpha) .
$$

Similarly,

$$
\begin{aligned}
\mathrm{e}^{-\lambda t}\left\|A^{\gamma} v(t)\right\|_{L^{2}(\Omega)} & \leqslant c \int_{0}^{t} \mathrm{e}^{-\lambda(t-s)}\left\|A^{\gamma} E(t-s)\right\|\|q\|_{L^{2}(\Omega)} \mathrm{e}^{-\lambda s}\|f(s)\| C\left([0, T] ; D\left(A^{\gamma}\right)\right) \\
& \mathrm{d} s \\
& \leqslant c \int_{0}^{t} \mathrm{e}^{-\lambda s} s^{(1-\gamma) \alpha-1} \mathrm{~d} s\|q\|_{L^{2}(\Omega)}\|f\|_{\lambda} \leqslant c \lambda^{-(1-\gamma) \alpha}\|q\|_{L^{2}(\Omega)}\|f\|_{\lambda} .
\end{aligned}
$$

Meanwhile, for $t \in[0, T)$ and $\tau>0$ with $t+\tau \leqslant T$, we have

$$
\begin{aligned}
A^{\gamma}(v(t+\tau)-v(t))= & \int_{0}^{t} A^{\gamma} E(s) q[f(t+\tau-s)-f(t-s)] \mathrm{d} s \\
& +\int_{t}^{t+\tau} A^{\gamma} E(s) q f(t+\tau-s) \mathrm{d} s,
\end{aligned}
$$

which directly implies

$$
\begin{aligned}
& \mathrm{e}^{-\lambda(t+\tau+1)}\left\|A^{\gamma}(v(t+\tau)-v(t))\right\|_{L^{2}(\Omega)} \\
& \leqslant c \int_{0}^{t} \mathrm{e}^{-\lambda s}\left\|A^{\gamma} E(s)\right\|\|q\|_{L^{2}(\Omega)} \mathrm{e}^{-\lambda(t+\tau-s+1)} \\
& \quad \times\|f(t+\tau-s)-f(t-s)\|_{D\left(A^{\gamma}\right)} \mathrm{d} s+c \int_{t}^{t+\tau} \mathrm{e}^{-\lambda(s+1)}\left\|A^{\gamma} E(s)\right\| \\
& \quad \times\|q\|_{L^{2}(\Omega)} \mathrm{e}^{-\lambda(t+\tau-s)}\|f(t+\tau-s)\|_{D(A \gamma)} \mathrm{d} s \\
& \leqslant \\
& \quad c \tau^{\beta}\|q\|_{L^{2}(\Omega)}\|f\|_{\lambda}\left(\int_{0}^{t} \mathrm{e}^{-\lambda s} s^{(1-\gamma) \alpha-1} \mathrm{~d} s+\tau^{-\beta} \int_{t}^{t+\tau} \mathrm{e}^{-\lambda(s+1)} s^{(1-\gamma) \alpha-1} \mathrm{~d} s\right) .
\end{aligned}
$$

This, the inequality (2.3) and the choice $\beta<(1-\gamma) \alpha$ give

$$
\begin{aligned}
& \mathrm{e}^{-\lambda(t+\tau+1)} \tau^{-\beta}\left\|A^{\gamma}(v(t+\tau)-v(t))\right\|_{L^{2}(\Omega)} \\
& \quad \leqslant c\|q\|_{L^{2}(\Omega)}\|f\|_{\lambda}\left(\lambda^{-(1-\gamma) \alpha}+\tau^{(1-\lambda) \alpha-\beta} \mathrm{e}^{-\lambda}\right) \leqslant c\|q\|_{L^{2}(\Omega)}\|f\|_{\lambda}\left(\lambda^{-(1-\gamma) \alpha}+\mathrm{e}^{-\lambda}\right) .
\end{aligned}
$$

In the same way, we deduce

$$
\tau^{-\beta} \mathrm{e}^{-\lambda(t+\tau+1)}\|v(t+\tau)-v(t)\|_{L^{2}(\Omega)} \leqslant c_{T}\|q\|_{L^{2}(\Omega)}\|f\|_{\lambda}\left(\lambda^{-\alpha}+\mathrm{e}^{-\lambda}\right) .
$$


Combining the preceding two estimates gives

$$
\sup _{0 \leqslant s<t \leqslant T} \mathrm{e}^{-\lambda(t+1)} \frac{\|v(t)-v(s)\|_{D\left(A^{\gamma}\right)}}{|t-s|^{\beta}} \leqslant c_{T}\|q\|_{L^{2}(\Omega)}\|f\|_{\lambda}\left(\lambda^{-(1-\gamma) \alpha}+\mathrm{e}^{-\lambda}\right) .
$$

Next, in view of the identities $\frac{\mathrm{d}}{\mathrm{d} t}(I-F(t))=A E(t)[17]$ and $\lim _{t \rightarrow 0^{+}}\|F(t)-I\|=0$, we deduce

$$
-A v(t)=\int_{0}^{t}-A E(t-s) q[f(s)-f(t)]+F(t) q f(t)-q f(t) .
$$

Then the preceding argument and lemma 2.1 lead to

$$
\begin{aligned}
\mathrm{e}^{-\lambda(t+2)}\|A v(t)\|_{L^{2}(\Omega)} \leqslant & c \int_{0}^{t} \mathrm{e}^{-\lambda}\|A E(t-s)\|\|q\|_{L^{2}(\Omega)} \\
& \times \mathrm{e}^{-\lambda(t+1)}\|f(s)-f(t)\|_{D(A \gamma)} \mathrm{d} s \\
& +c \mathrm{e}^{-\lambda(t+2)}\|q\|_{L^{2}(\Omega)}\|f(t)\|_{D(A \gamma)} \\
\leqslant & c\|q\|_{L^{2}(\Omega)}\|f\|_{\lambda} \mathrm{e}^{-\lambda}\left(\int_{0}^{t}(t-s)^{\beta-1} \mathrm{~d} s+1\right) \\
\leqslant & c_{T}\|q\|_{L^{2}(\Omega)}\|f\|_{\lambda} \mathrm{e}^{-\lambda} .
\end{aligned}
$$

Combining the preceding estimates implies

$$
\|L(q) f\|_{\lambda} \leqslant c_{T}\left(\mathrm{e}^{-\lambda}+\lambda^{-(1-\gamma) \alpha}\right)\|q\|_{L^{2}(\Omega)}\|f\|_{\lambda} .
$$

It follows directly from this estimate that the function $\|L(q) f\|_{\lambda}$ tends to zero as $\lambda$ tends to infinity, and thus the operator norm $\|L(q)\|_{\lambda}<1$ if $\lambda$ is large enough, which shows the lemma.

Now we can state the unique solvability of the Cauchy problem (2.1).

Proposition 2.1. If $q \in L^{2}(\Omega)$ and $u_{0} \in D\left(A^{1+\gamma}\right)$. Then the Cauchy problem (2.1) has a unique classical solution $u(q)=(I-L(q))^{-1} U$.

Proof. Since $u_{0} \in D\left(A^{1+\gamma}\right)$, by the regularity theory for subdiffusion [35, theorems 2.1 and 2.3], $U \in X$. Thus, by lemma 2.3, the Cauchy problem (2.1) has a unique solution $u=(I-$ $L(q))^{-1} U \in X$. The fact that $u$ is the classical solution to problem (2.1) follows from the fact that $q u \in C^{\beta}\left([0, T] ; L^{2}(\Omega)\right)$ and $u_{0} \in D(A)$, by the regularity theory of subdiffusion [35].

\section{Proof of theorem 1.1}

Below we assume $u_{0}$ satisfies the condition of theorem 1.1. In view of the Sobolev embed$\operatorname{ding} D\left(A^{1+\gamma}\right) \hookrightarrow C^{2, \delta}(\bar{\Omega})$ for some $\delta>0$, the function $U(t) \in C^{2+\delta, \frac{2+\delta}{2} \alpha}(\bar{\Omega} \times[0, T])[24]$, and satisfies

$$
\left\{\begin{aligned}
\partial_{t}^{\alpha} U & =\Delta U, \quad \text { in } \Omega \times(0, T], \\
U(0) & =u_{0}, \quad \text { in } \Omega, \\
U & =0, \quad \text { on } \partial \Omega \times[0, T] .
\end{aligned}\right.
$$

The next result collect several properties of the function $U(t)$.

Lemma 3.1. The following properties hold on the function $U(t)$. 
(a) $\mu_{0} \bar{\varphi}_{1}(x) \leqslant u_{0}(x) \leqslant \mu_{1} \bar{\varphi}_{1}(x), x \in \bar{\Omega}$.

(b) $\mu_{0} E_{\alpha, 1}\left(-\lambda_{1} t^{\alpha}\right) \bar{\varphi}_{1}(x) \leqslant U(x, t) \leqslant \mu_{1} E_{\alpha, 1}\left(-\lambda_{1} t^{\alpha}\right) \bar{\varphi}_{1}(x),(x, t) \in \bar{\Omega} \times[0, T]$.

(c) $0 \leqslant-\partial_{t}^{\alpha} U(x, t) \leqslant\left\|\Delta u_{0}\right\|_{L^{\infty}(\Omega)} \leqslant \mu_{1} \lambda_{1},(x, t) \in \Omega \times[0, T]$

Proof. Part (a) is already proved in [6]. Part (b) follows from the maximum principle for the subdiffusion model (see, e.g., [27, Theorem 1.1] or [28]). We only prove (c). Let $w(x, t)=\partial_{t}^{\alpha} U(x, t)$. Then $w$ satisfies

$$
\left\{\begin{aligned}
\partial_{t}^{\alpha} w & =\Delta w, & & \text { in } \Omega \times(0, T], \\
w(0) & =\Delta u_{0}, & & \text { in } \Omega, \\
w & =0, & & \text { on } \partial \Omega \times[0, T] .
\end{aligned}\right.
$$

By assumption, $\Delta u_{0} \leqslant 0$ in $\Omega$, and thus by the maximum principle for the subdiffusion [27, theorem 1.1], $0 \leqslant-w(x, t) \leqslant\left\|\Delta u_{0}\right\|_{L^{\infty}(\Omega)}$. This implies assertion (c).

Let $\omega$ be defined as in theorem 1.1. Lemma 3.1(b) implies that

$$
u_{T}=\frac{1}{\left.U(T)\right|_{\omega}}
$$

extended by zero outside $\omega$ belongs to $L^{\infty}(\Omega)$. Now we define the operator $P_{T}: L^{2}(\omega) \rightarrow L^{2}(\omega)$ by

$$
q \mapsto \int_{0}^{T}-A E(T-s) u_{T}[U(s)-U(T)] q \mathrm{~d} s+F(T) q .
$$

This operator arises in the linearization of the forward map.

The next result gives an upper bound on the constant $c_{\alpha}$ defined in (1.3). In particular, it indicates that $c_{\alpha}<\alpha<1$, which is crucial for proving theorem 1.1.

Proposition 3.1. For any $\alpha \in(0,1)$,

$$
c_{\alpha}:=\sup _{t \geqslant 0} t E_{\alpha, \alpha}(-t) \leqslant \frac{\alpha^{2} \pi}{\sin (\alpha \pi)+\alpha \pi} .
$$

Proof. Let $f(t)=t E_{\alpha, \alpha}(-t)$. By the asymptotics of the Mittag-Leffler function $E_{\alpha, \alpha}(-t)$, i.e., $E_{\alpha, \alpha}(-t) \leqslant \frac{c}{1+t^{2}}$, and complete monotonicity of the function $E_{\alpha, \alpha}(-t)$, the function $f(t)$ is nonnegative on $[0, \infty)$, and tends to zero as $t \rightarrow \infty$. Thus, there exists a maximum. Now let $u(t)=t^{\alpha-1} E_{\alpha, \alpha}\left(-t^{\alpha}\right)$. Then it satisfies the following ODE

$$
{ }^{R} \partial_{t}^{\alpha} u+u=0, \quad t>0, \quad \text { with } \quad{ }^{0} I_{t}^{1-\alpha} u(0)=1,
$$

where the notation ${ }^{R} \partial_{t}^{\alpha}$ and ${ }^{0} I_{t}^{\beta}$ denote the Riemann-Liouville fractional derivative and integral, respectively, based at $t=0$. Let $w(t)=t u(t)=t^{\alpha} E_{\alpha, \alpha}\left(-t^{\alpha}\right)$. Then direct computation with the identity ${ }^{R} \partial_{t}^{\alpha} t^{\gamma}=\frac{\Gamma(\gamma+1)}{\Gamma(\gamma-\alpha+1)} t^{\gamma-\alpha}$ leads to

$$
{ }^{R} \partial_{t}^{\alpha} w(t)=\sum_{k=0}^{\infty} \frac{(-1)^{k}}{\Gamma(k \alpha+\alpha)}{ }^{R} \partial_{t}^{\alpha} t^{k \alpha+\alpha}=\sum_{k=0}^{\infty} \frac{(-1)^{k}}{\Gamma(k \alpha+\alpha)} \frac{\Gamma(k \alpha+\alpha+1)}{\Gamma(k \alpha+1)} t^{k \alpha} .
$$


Using the recursion $\Gamma(z+1)=z \Gamma(z)$ twice,

$$
\begin{aligned}
{ }^{R} \partial_{t}^{\alpha} w(t) & =\sum_{k=0}^{\infty} \frac{k \alpha(-1)^{k}}{\Gamma(k \alpha+1)} t^{k \alpha}+\alpha \sum_{k=0}^{\infty} \frac{(-1)^{k}}{\Gamma(k \alpha+1)} t^{k \alpha} \\
& =\sum_{k=0}^{\infty} \frac{(-1)^{k}}{\Gamma(k \alpha)} t^{k \alpha}+\alpha \sum_{k=0}^{\infty} \frac{(-1)^{k}}{\Gamma(k \alpha+1)} t^{k \alpha} \\
& =-t^{\alpha} E_{\alpha, \alpha}\left(-t^{\alpha}\right)+\alpha E_{\alpha, 1}\left(-t^{\alpha}\right),
\end{aligned}
$$

where the last step follows since $1 / \Gamma(0)=0$. Consequently,

$$
{ }^{R} \partial_{t}^{\alpha} w+w=\alpha E_{\alpha, 1}\left(-t^{\alpha}\right) \quad \text { with }{ }^{0} I_{t}^{1-\alpha} w(0)=0 .
$$

Thus, the solution theory for fractional ODEs indicates that $w(t)$ is represented by

$$
w(t)=\int_{0}^{t}(t-s)^{\alpha-1} E_{\alpha, \alpha}\left(-(t-s)^{\alpha}\right) \alpha E_{\alpha, 1}\left(-s^{\alpha}\right) \mathrm{d} s .
$$

Then using the facts that $0 \leqslant E_{\alpha, 1}(-t) \leqslant 1, E_{\alpha, \alpha}(-t) \geqslant 0$ (as a result of the complete monotonicity of $\left.E_{\alpha, 1}(-t)[32]\right)$, and the differentiation formula

$$
\frac{\mathrm{d}}{\mathrm{d} t} E_{\alpha, 1}\left(-t^{\alpha}\right)=-t^{\alpha-1} E_{\alpha, \alpha}\left(-t^{\alpha}\right)
$$

we deduce

$$
w(t) \leqslant \alpha \int_{0}^{t}(t-s)^{\alpha-1} E_{\alpha, \alpha}\left(-(t-s)^{\alpha}\right) \mathrm{d} s=\alpha\left(1-E_{\alpha, 1}\left(-t^{\alpha}\right)\right)<\alpha .
$$

Meanwhile, by Simon's theorem [38],

$$
E_{\alpha, 1}\left(-t^{\alpha}\right) \leqslant \frac{1}{1+\Gamma(1+\alpha)^{-1} t^{\alpha}}<\Gamma(1+\alpha) t^{-\alpha}
$$

there holds

$$
\begin{aligned}
w(t) & =\int_{0}^{t}(t-s)^{\alpha-1} E_{\alpha, \alpha}\left(-(t-s)^{\alpha}\right) \alpha E_{\alpha, 1}\left(-s^{\alpha}\right) \mathrm{d} s \\
& \leqslant \alpha \Gamma(\alpha+1) \int_{0}^{t}(t-s)^{\alpha-1} E_{\alpha, \alpha}\left(-(t-s)^{\alpha}\right) s^{-\alpha} \mathrm{d} s \\
& =\alpha \Gamma(\alpha+1) \int_{0}^{t} \sum_{k=0}^{\infty} \frac{(-1)^{k}}{\Gamma(k \alpha+\alpha)}(t-s)^{k \alpha+\alpha-1} s^{-\alpha} \mathrm{d} s .
\end{aligned}
$$

Using the identity $\int_{0}^{t}(t-s)^{a-1} s^{-b} \mathrm{~d} s=t^{a-b} \frac{\Gamma(a) \Gamma(1-b)}{\Gamma(a+1-b)}$ for any $a>0$ and $b<1$, we deduce

$$
\begin{aligned}
w(t) & \leqslant \alpha \Gamma(\alpha+1) \sum_{k=0}^{\infty} \frac{(-1)^{k}}{\Gamma(k \alpha+\alpha)} \frac{\Gamma(k \alpha+\alpha) \Gamma(1-\alpha)}{\Gamma(k \alpha+1)} t^{k \alpha} \\
& \leqslant \alpha \Gamma(\alpha+1) \Gamma(1-\alpha) \sum_{k=0}^{\infty} \frac{(-1)^{k}}{\Gamma(k \alpha+1)} t^{k \alpha}=\alpha \Gamma(1-\alpha) \Gamma(1+\alpha) E_{\alpha, 1}\left(-t^{\alpha}\right) .
\end{aligned}
$$


Now by the recursion identity and reflection identity for the Gamma function,

$$
\Gamma(1-\alpha) \Gamma(1+\alpha)=\alpha \Gamma(1-\alpha) \Gamma(\alpha)=\frac{\alpha \pi}{\sin (\alpha \pi)} .
$$

Combining the preceding estimates leads directly to

$$
\sup _{t \geqslant 0} t E_{\alpha, \alpha}(-t) \leqslant \alpha \max _{t \geqslant 0} \min \left(\frac{\alpha \pi}{\sin (\alpha \pi)} E_{\alpha, 1}\left(-t^{\alpha}\right), 1-E_{\alpha, 1}\left(-t^{\alpha}\right)\right) .
$$

By the complete monotonicity of $E_{\alpha, 1}(-t)$, the first term $\frac{\alpha \pi}{\sin (\alpha \pi)} E_{\alpha, 1}\left(-t^{\alpha}\right)$ in the bracket is monotonically decreasing, whereas the second term $1-E_{\alpha, 1}\left(-t^{\alpha}\right)$ is monotonically increasing. Thus, one simple upper bound is obtained by equating these two terms, which directly gives

$$
E_{\alpha, 1}\left(-t_{*}^{\alpha}\right)=\frac{1}{1+\frac{\alpha \pi}{\sin (\alpha \pi)}}
$$

Upon substituting it back to (3.2) and noting the complete monotonicity of $E_{\alpha, 1}(-t)$, we deduce

$$
c_{\alpha} \leqslant \alpha \frac{\frac{\alpha \pi}{\sin (\alpha \pi)}}{1+\frac{\alpha \pi}{\sin (\alpha \pi)}}=\frac{\alpha^{2} \pi}{\alpha \pi+\sin (\alpha \pi)} .
$$

This completes the proof of the proposition.

Remark 3.1. Note that the identities

$$
\lim _{\alpha \rightarrow 0^{+}} \frac{\alpha \pi}{\alpha \pi+\sin \alpha \pi}=\frac{1}{2} \text { and } \lim _{\alpha \rightarrow 1^{-}} \frac{\alpha \pi}{\alpha \pi+\sin \alpha \pi}=1 .
$$

and the function $f(\alpha)=\frac{\alpha \pi}{\alpha \pi+\sin \alpha \pi}$ is strictly increasing in $\alpha$ over the interval $(0,1)$. Thus, the factor is strictly less than 1 for any $\alpha \in(0,1)$. Note also that for the limiting case $\alpha=1$, the constant $c_{1}=\sup _{t \geqslant 0} t \mathrm{e}^{-t}=\mathrm{e}^{-1}$, which is much sharper than the preceding bound. Since the function $E_{\alpha, \alpha}(-t)$ is actually continuous in $\alpha$, one may refine the bound on $c_{\alpha}$ slightly for $\alpha$ close to unit. Further, it is worth noting that the integral representation (3.1) for $w(t)$ can also be deduced from the following Christoffel-Darboux type formula for Mittag-Leffler functions, i.e.,

$$
\begin{array}{r}
\int_{0}^{t} s^{\gamma-1} E_{\alpha, \gamma}\left(y s^{\alpha}\right)(t-s)^{\beta-1} E_{\alpha, \beta}\left(z(t-s)^{\alpha}\right) \mathrm{d} s \\
=\frac{y E_{\alpha, \gamma+\beta}\left(y t^{\alpha}\right)-z E_{\alpha, \gamma+\beta}\left(z t^{\alpha}\right)}{y-z} t^{\gamma+\beta-1},
\end{array}
$$

where $y \neq z$ are any complex numbers. Consequently, by a limiting argument,

$$
\int_{0}^{t} s^{\alpha-1} E_{\alpha, \alpha}\left(-s^{\alpha}\right) E_{\alpha, 1}\left(-(t-s)^{\alpha}\right) \mathrm{d} s=\left.\frac{\mathrm{d}}{\mathrm{d} \lambda} \lambda t^{\alpha} E_{\alpha, \alpha+1}\left(\lambda t^{\alpha}\right)\right|_{\lambda=-1},
$$

which upon simplification gives directly the formula (3.1) for $w(t)$.

Remark 3.2. Proposition 3.1 provides an upper bound on the constant $c_{\alpha}$. In figure 1(a), we plot the function $\alpha^{-1} t E_{\alpha, \alpha}(-t)$ versus $t$ for several different fractional orders, where the Mittag-Leffler function $E_{\alpha, \alpha}(-t)$ is computed using an algorithm developed in [37]. Clearly, for any fixed $\alpha$, the function $t E_{\alpha, \alpha}(-t)$ first increases with $t$ and then decreases, and there is only one global maximum. The maximum is always achieved at some $t^{*}$ between 0.8 and 1 , a fact that remains to be established, and the maximum value decreases with $\alpha$. The optimal 

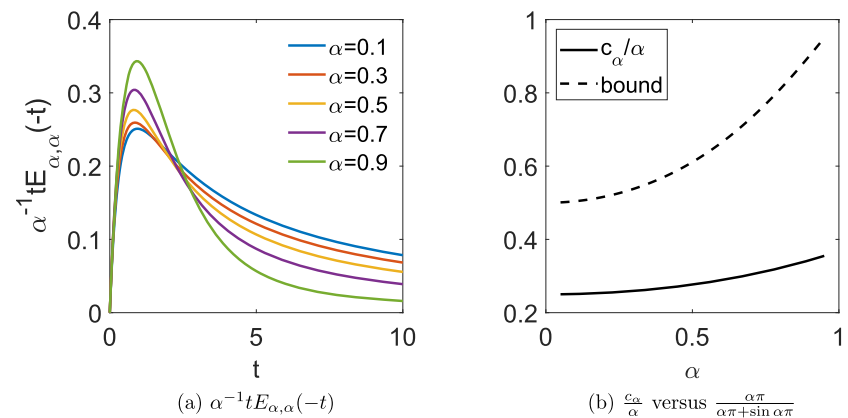

Figure 1. The function $\alpha^{-1} t E_{\alpha, \alpha}(-t)$ and its maximum $\frac{c_{\alpha}}{\alpha}$ versus the upper bound $\frac{\alpha \pi}{\alpha \pi+\sin \alpha \pi}$ in proposition 3.1

constant $\frac{c_{\alpha}}{\alpha}$ versus the upper bound $\frac{\alpha \pi}{\sin \alpha \pi+\alpha \pi}$ is shown in figure 1(b). Note that $\frac{c_{\alpha}}{\alpha}$ is strictly increasing with respect to $\alpha$, and the upper bound in proposition 3.1 is about three times larger than the optimal one $\frac{c_{\alpha}}{\alpha}$. This is attributed to the fact that the derivation employs upper bounds of the Mittag-Leffler function $E_{\alpha, 1}(-t)$ that are valid on the entire real line, instead of sharper ones on a finite interval, e.g., $[0,1]$. The fact that the ratio $\frac{c_{\alpha}}{\alpha}$ increases with $\alpha$ implies that the smaller the fractional order $\alpha$ is, there is a larger degree of freedom for choosing the parameter $\epsilon$ as well as $\mu_{1} / \mu_{0}$ in theorem 1.1 , which partly indicates the potential beneficial effect of subdiffusion on the inverse potential problem.

The next result gives the invertibility of the operator $I-P_{T}$ on $L^{2}(\omega)$.

Lemma 3.2. Under the assumptions of theorem 1.1, there exists a $\theta>0$ depending only on $\alpha$ and $\epsilon$ such that if $\lambda_{1} T^{\alpha}<\theta$, then the operator $I-P_{T}$ has a bounded inverse in $B\left(L^{2}(\omega)\right)$.

Proof. First, we bound $\|t A E(t)\|$. Using the eigenpairs $\left\{\left(\lambda_{j}, \varphi_{j}\right)\right\}_{j=1}^{\infty}$ of the operator $A$, we deduce

$$
E(t) v=\sum_{j=1}^{\infty} t^{\alpha-1} E_{\alpha, \alpha}\left(-\lambda_{j} t^{\alpha}\right)\left(\varphi_{j}, v\right) \varphi_{j}, \quad \forall v \in L^{2}(\Omega)
$$

Thus,

$$
\|t A E(t) v\|^{2}=\sum_{j=1}^{\infty}\left(\lambda_{j} t^{\alpha} E_{\alpha, \alpha}\left(-\lambda_{j} t^{\alpha}\right)\right)^{2}\left(v, \varphi_{j}\right)^{2} .
$$

Since $\sup _{t \in[0, \infty]}\left|t E_{\alpha, \alpha}(-t)\right| \leqslant c_{\alpha}<\alpha$, in view of proposition 3.1,

$$
\|A E(t) v\| \leqslant c_{\alpha} t^{-1} .
$$

Meanwhile, using the governing equation for $U(t)$, we have

$$
U(t)-U(0)={ }^{0} I_{t}^{\alpha} \Delta U(t),
$$

which together with the fact $\Delta U(x, t) \leqslant 0$ implies

$$
\begin{aligned}
U(t)-U(T) & =\left({ }^{0} I_{t}^{\alpha} \Delta U\right)(t)-\left({ }^{0} I_{t}^{\alpha} \Delta U\right)(T) \\
& =\frac{1}{\Gamma(\alpha)} \int_{0}^{t}\left[(T-s)^{\alpha-1}-(t-s)^{\alpha-1}\right](-\Delta U(s)) \mathrm{d} s+\frac{1}{\Gamma(\alpha)} \int_{t}^{T}(T-s)^{\alpha-1}(-\Delta U(s)) \mathrm{d} s .
\end{aligned}
$$


Since $(T-s)^{\alpha-1}-(t-s)^{\alpha-1} \leqslant 0$ and $-\Delta U(x, t) \geqslant 0$ in $\Omega \times[0, T]$, by lemma 3.1(c)

$$
U(t)-U(T) \leqslant \frac{1}{\Gamma(\alpha)} \int_{t}^{T}(T-s)^{\alpha-1}(-\Delta U(s)) \mathrm{d} s \leqslant \frac{(T-t)^{\alpha}}{\Gamma(\alpha+1)} \mu_{1} \lambda_{1}
$$

Similarly,

$$
\begin{aligned}
U(T)-U(t) & \leqslant \frac{1}{\Gamma(\alpha)} \int_{0}^{t}\left[(t-s)^{\alpha-1}-(T-s)^{\alpha-1}\right](-\Delta U(s)) \mathrm{d} s \\
& \leqslant \frac{\mu_{1} \lambda_{1}}{\Gamma(\alpha+1)}\left(t^{\alpha}+(T-t)^{\alpha}-T^{\alpha}\right) \leqslant \frac{(T-t)^{\alpha}}{\Gamma(\alpha+1)} \mu_{1} \lambda_{1}
\end{aligned}
$$

Consequently, there holds

$$
\|U(s)-U(T)\|_{L^{\infty}(\Omega)} \leqslant \frac{1}{\Gamma(\alpha+1)} \mu_{1} \lambda_{1}(T-s)^{\alpha} .
$$

Lemma 3.1(b) implies

$$
\left\|u_{T}\right\|_{L^{\infty}(\Omega)} \leqslant \frac{1}{\mu_{0}(1-\epsilon) E_{\alpha, 1}\left(-\lambda_{1} T^{\alpha}\right)} .
$$

The preceding two estimates and lemma 2.1 imply

$$
\begin{aligned}
\left\|P_{T}\right\|_{B\left(L^{2}(\omega)\right)} \leqslant & \int_{0}^{T}\|A E(T-s)\|\left\|u_{T}\right\|_{L^{\infty}(\Omega)}\|U(s)-U(T)\|_{L^{\infty}(\Omega)} \mathrm{d} s+\|F(T)\| \\
\leqslant & \int_{0}^{T} c_{\alpha}(T-s)^{-1} \frac{\lambda_{1} \mu_{1}}{\Gamma(\alpha+1)}(T-s)^{\alpha} \frac{1}{\mu_{0}(1-\epsilon) E_{\alpha, 1}\left(-\lambda_{1} T^{\alpha}\right)} \mathrm{d} s \\
& +E_{\alpha, 1}\left(-\lambda_{1} T^{\alpha}\right) \\
= & \frac{c_{\alpha} \mu_{1}}{\mu_{0}(1-\epsilon) \alpha \Gamma(\alpha+1) E_{\alpha, 1}\left(-\lambda_{1} T^{\alpha}\right)} \lambda_{1} T^{\alpha}+E_{\alpha, 1}\left(-\lambda_{1} T^{\alpha}\right) .
\end{aligned}
$$

Let $m(x)$ be defined by

$$
m(x)=\frac{c_{\alpha} \mu_{1}}{\mu_{0}(1-\epsilon) \alpha \Gamma(\alpha+1)} \frac{x}{E_{\alpha, 1}(-x)}+E_{\alpha, 1}(-x) .
$$

Straightforward computation shows

$$
m^{\prime}(x)=\frac{c_{\alpha} \mu_{1}}{\mu_{0}(1-\epsilon) \alpha \Gamma(\alpha+1)} \frac{E_{\alpha, 1}(-x)-x E_{\alpha, 1}^{\prime}(-x)}{E_{\alpha, 1}(-x)^{2}}+E_{\alpha, 1}^{\prime}(-x) .
$$

Thus, $m(0)=1$ and by proposition 3.1 ,

$$
m^{\prime}(0)=\frac{c_{\alpha} \mu_{1}}{\mu_{0}(1-\epsilon) \alpha \Gamma(\alpha+1)}-\frac{1}{\Gamma(\alpha+1)}=\left[\frac{c_{\alpha} \mu_{1}}{\mu_{0}(1-\epsilon) \alpha}-1\right] \frac{1}{\Gamma(\alpha+1)}<0,
$$

under the given conditions on $\epsilon, \mu_{0}$ and $\mu_{1}$ in theorem 1.1. Thus, there exists a $\theta>0$ such that whenever $x<\theta, m(x)<1$, and accordingly, for $\lambda_{1} T^{\alpha}$ sufficiently close to zero, $P_{T}$ is a contraction on $L^{2}(\omega)$. Then by Neumann series expansion, $I-P_{T}$ is invertible and $\left(I-P_{T}\right)^{-1}$ is bounded. This completes the proof of the lemma. 
Now we introduce the trace operator: $\operatorname{tr}: X \rightarrow D(A), v \mapsto v(T)$. Then $\operatorname{tr} \in B(X, D(A))$ and $\|\operatorname{tr}\|_{B(X, D(A))} \leqslant 1$. Finally, we can present the proof of theorem 1.1.

Proof. With lemma 3.2 at hand, the proof is identical with that of [6]. We only include a proof for the convenience of readers. We define the mapping: $K: L^{2}(\omega) \rightarrow L^{2}(\omega)$, $\left.q \mapsto[-A u(q)(T)]\right|_{\omega}=\left.\left[-A \operatorname{tr}(I-L(q))^{-1} U\right]\right|_{\omega}$. Clearly, $K$ is continuously Fréchet differentiable, cf lemma 2.3, and its derivative $K^{\prime}$ at $q \in L^{2}(\omega)$ in the direction $p$ is given by

$$
K^{\prime}(q)[p]=\left.\left[-A \operatorname{tr}(I-L(q))^{-1} L(p)(I-L(q))^{-1} U\right]\right|_{\omega} .
$$

Let $Q_{T}=K^{\prime}(0)=\left.[-A \operatorname{tr} L(\cdot) U]\right|_{\omega}$. Then

$$
Q_{T}(p)=\left[\int_{0}^{T}-A E(T-s) p[U(s)-U(T)] \mathrm{d} s+(F(T)-I) p U(T)\right] k_{\omega} .
$$

We define a multiplication operator $M: L^{2}(\omega) \rightarrow L^{2}(\omega), p \rightarrow U(T) p$. Then $M$ is invertible, and its inverse is exactly the multiplication operator by $u_{T}$. Consequently, $Q_{T} M^{-1}=P_{T}-I$. By lemma 3.2, $\left(P_{T}-I\right)^{-1}$ belongs to $B\left(L^{2}(\omega)\right)$. Therefore, $Q_{T}$ has a bounded inverse and $Q_{T}^{-1}=M^{-1}\left(P_{T}-I\right)^{-1}$. By the implicit function theorem, $K$ is locally a $C^{1}$-diffeomorphism from a neighborhood of 0 onto a neighborhood of $K(0)$. In particular, $K^{-1}$ is Lipschitz continuous in a neighborhood of $K(0)$. Then theorem 1.1 follows by noting the following inequality

$$
\left\|\left.A u\left(q_{1}\right)(T)\right|_{\omega}-\left.A u\left(q_{2}\right)(T)\right|_{\omega}\right\|_{L^{2}(\omega)} \leqslant\left\|u\left(q_{1}\right)(T)-u\left(q_{2}\right)(T)\right\|_{D(A)},
$$

for any $q_{1}, q_{2} \in L^{2}(\omega)$.

\section{Fixed point algorithm}

Now we propose a simple fixed point algorithm to find the potential $q$ from the terminal observation. Given a noisy version of the exact data $g=u\left(q^{\dagger}\right)(T)$ corresponding to the exact potential $q^{\dagger}$ and an initial guess $q^{0}$, we employ the following fixed point iteration

$$
q^{k+1}=F\left(q^{k}\right), \quad \text { with } F(q)=q+\lambda A^{-1}(u(q)(T)-g),
$$

where $\lambda>0$ is a relaxation parameter and $A=-\Delta$ is the negative Laplacian with a zero Dirichlet boundary condition. In the absence of the preconditioning operator $A^{-1}$, the iteration (4.1) was proposed in [34] for the standard parabolic problem. For both normal diffusion and subdiffusion, the unpreconditioned version works very robustly for exact data, but it tends to suffer from severe numerical instability in the presence of data noise. This is attributed to the fact that the noise in the data $g$ is amplified by a factor $\lambda$ at each iteration, in view of the smoothing property of the solution operator, and the noise effect accumulates very rapidly so as to completely spoil the reconstruction after a few iterations. The preconditioner $A^{-1}$ is to mitigate the deleterious effect of noise in the observation $g$ by implicitly filtering out the highfrequency components present in the noise thereby achieving a form of regularization [9]. Numerically, the scheme is straightforward to implement since it requires only one forward solve, and the preconditioning step incurs very little extra computational effort.

We have the following contractive property on the cone $S=\{h \in C(\bar{\Omega}): h(x) \geqslant 0\}$.

Proposition 4.1. For any nonnegative $u_{0} \not \equiv 0$ and any $q \in S$, the linearized map $F^{\prime}$ is contractive on $\mathrm{S}$ in the following sense

$$
\left\|F^{\prime}(q) h\right\|_{L^{2}(\Omega)}<\|h\|_{L^{2}(\Omega)}, \quad \forall h \in S,
$$


provided that the relaxation parameter $\lambda$ is sufficiently small.

Proof. For any $q, h \in S$, the Gâteaux derivative $F$ is given by

$$
F^{\prime}(q)[h]=h-\lambda A^{-1} v(T),
$$

where $v \equiv v(q, h)$ satisfies the following inhomogeneous problem

$$
\left\{\begin{aligned}
\partial_{t}^{\alpha} v & =\Delta v-q v+h u(q), & & \text { in } \Omega \times(0, T], \\
v & =0, & & \text { on } \partial \Omega \times(0, T], \\
v(0) & =0, & & \text { in } \Omega .
\end{aligned}\right.
$$

By the 'strong' maximum principle for the subdiffusion model [28] and the nonnegativity of $u_{0}$ and $q$ that

$$
0<u(q)(x, t) \leqslant\left\|u_{0}\right\|_{L^{\infty}(\Omega)} \quad \forall(x, t) \in \Omega \times(0, T] .
$$

Since $h \in S$, the maximum principle [28] shows $v(x, t) \geqslant$ in $\Omega \times[0, T]$. Further, with $f(t)=h u(t)$, the solution $v$ can be represented by

$$
v(t)=\int_{0}^{t} E(t-s) f(s) \mathrm{d} s=\sum_{j=1}^{\infty} \int_{0}^{t}(t-s)^{\alpha-1} E_{\alpha, \alpha}\left(-\lambda_{j}(t-s)^{\alpha}\right)\left(f(s), \varphi_{j}\right) \mathrm{d} s \varphi_{j} .
$$

In particular,

$$
\left(v(T), \varphi_{1}\right)=\int_{0}^{t}(t-s)^{\alpha-1} E_{\alpha, \alpha}\left(-\lambda_{1}(t-s)^{\alpha}\right)\left(f(s), \varphi_{1}\right) \mathrm{d} s .
$$

Now if $h \not \equiv 0$, then for any fixed $t>0, \operatorname{supp}(f(t))=\operatorname{supp}(h)$, and by the positivity of $\varphi_{1}$ in $\Omega$, $\left(f(t), \varphi_{1}\right)>0$ for any $t>0$. Thus, $0 \leqslant v(T) \not \equiv 0$, and by the properties of elliptic problems, $A^{-1} v(T)>0$ in $\Omega$. Thus, by choosing $\lambda$ sufficiently small (depending on $h$ ), we deduce the desired assertion.

The next result shows that the fixed point iteration (4.1) can actually also be interpreted as a preconditioned gradient descent method, under certain restrictions on $u_{0}$ and the residual $u(q)(T)-g$. The descent property can be numerically observed in a more general case, which, however, remains to be proved.

Proposition 4.2. If $u_{0} \not \equiv 0$ is nonnegative and $u(q)(T)-g \not \equiv 0$ is not sign changing, then $A^{-1}(u(q)(T)-g)$ is a descent direction to the functional $J(q)=\frac{1}{2}\|u(q)(T)-g\|_{L^{2}(\Omega)}^{2}$.

Proof. Let $w \equiv w(q)$ solve the adjoint problem

$$
\begin{cases}{ }_{t}^{R} \partial_{T}^{\alpha} w=\Delta w-q w, & \text { in } \Omega \times(0, T], \\ w=0, & \text { on } \partial \Omega \times(0, T], \\ { }_{t}^{R} \partial_{T}^{\alpha-1} w(T)=u(q)(T)-g, & \text { in } \Omega,\end{cases}
$$

where the notation ${ }_{t}^{R} \partial_{T}^{\alpha} w$ denotes the right-sided Riemann-Liouville fractional derivative (based at $T$ ), and ${ }_{t}^{R} \partial_{T}^{\alpha-1} w(T)$ the Riemann-Liouville fractional integral of order $1-\alpha$. Further, using the solution operator $E_{q}$ associated with the $\Delta-q, w$ can be represented by

$$
w(t)=E_{q}(T-t)(u(q)(T)-g)=\int_{t}^{T} E_{q}(s-t)(u(q)(T)-g) \delta_{T}(s) \mathrm{d} s,
$$


Algorithm 1. Anderson acceleration for the fixed point iteration (4.1).

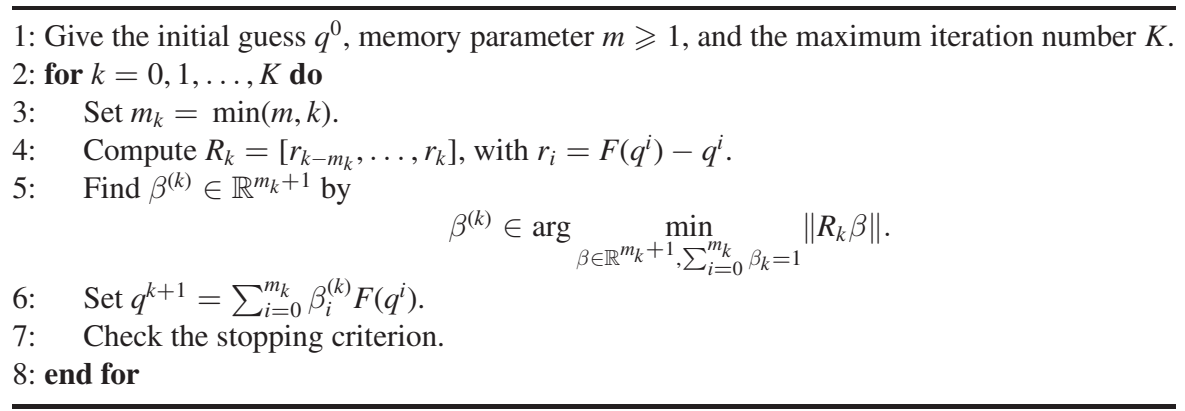

where $\delta_{T}(s)$ denotes the Dirac delta function at $T$. Then direct computation shows that the gradient $J^{\prime}(q)$ to the functional $J(q)$ is given by

$$
J^{\prime}(q)=-\int_{0}^{T} u(q) w(q) \mathrm{d} t .
$$

Now it follows that

$$
\int_{\Omega} J^{\prime}(q) A^{-1}(u(q)(T)-g) \mathrm{d} x=-\int_{0}^{T} \int_{\Omega} u(q) w(q) A^{-1}(u(q)(T)-g) \mathrm{d} x \mathrm{~d} t .
$$

By the maximum principle for elliptic problems, $u(q)(T)-g \lessgtr 0$ in $\Omega$ implies $A^{-1}(u(q)(T)-$ $g) \lessgtr 0$ in $\Omega$, and similarly, $u(q)$ is positive almost everywhere in $\Omega \times(0, T)$ for nonnegative $u_{0} \neq \equiv 0$ [28]. Meanwhile, in view of the representation (4.2), using a density argument (i.e., approximating the singular source $(u(q)(T)-g) \delta_{T}(s)$ with $(u(q)(T)-g) \phi_{n}(t)$, with $\phi_{n} \geqslant 0$ being smooth and $\phi_{n}(t) \rightarrow \delta_{T}(t)$ weakly; see [28] for relevant argument) and the weak maximum principle for subdiffusion, $u(q)(T)-g \lessgtr 0$ implies $w(t) \lessgtr 0$ almost everywhere in $\Omega \times(0, T)$. Consequently, we arrive at

$$
\int_{\Omega} J^{\prime}(q) A^{-1}(u(q)(T)-g) \mathrm{d} x<0
$$

i.e., $A^{-1}(u(q)(T)-g)$ is a descent direction to the functional $J(q)$.

Numerical experiments indicate that the convergence behavior of fixed point iteration (4.1) depends very much on the relaxation parameter $\lambda>0$ : if $\lambda$ is small, then it converges steadily but only slowly, whereas for large $\lambda$, the convergence may be unstable and suffers from large oscillations. In order to accelerate the convergence, we employ the classical Andersson acceleration technique [2], which can be viewed as a version of GMRES for nonlinear problems [40]; see the review [3] for other related extrapolation techniques. The complete procedure for Anderson acceleration is listed in algorithm 1 . The integer $m$ controls the number of memory terms used for the Anderson update. Thus, the acceleration step only involves simple algebraic manipulations, and the associated computational overhead is negligible. In our experiment below, $m=2$ represents a good choice. At line 7 of the algorithm, the stopping criterion of the iteration can employ the standard discrepancy principle, i.e.,

$$
k^{*}=\min _{k \geqslant 1}\left\{\left\|u\left(q^{k}\right)-g\right\|_{L^{2}(\Omega)} \leqslant \tau \delta\right\},
$$

where $\tau>1$ is the tolerance, and $\delta=\left\|g-u\left(q^{\dagger}\right)\right\|_{L^{2}(\Omega)}$ is the noise level. The discrepancy principle is a well established early stopping strategy for iterative regularization methods [9]. 
The fixed point algorithm and its accelerated variant exhibit a very similar behavior in practice, when noise is present in the data; see section 5 for numerical illustrations.

Despite the enormous empirical success, the global convergence of Anderson acceleration remains completely open, even for affine linear maps with fixed memory (the case of linear map with full memory is well known due to its connection with GMRES [40]). The local convergence of Anderson acceleration for contractive maps was studied recently in [10, 39]. However, these results do not apply to the inverse potential problem, since the associated map is not a contraction.

Remark 4.1. In the fixed point iteration (4.1), the update does not change the boundary condition of the initial guess $q^{0}$. Thus, it is implicitly assumed that the boundary condition is exactly known. Further, for $g \in L^{2}(\Omega)$, by the standard elliptic regularity result, the update increment $A^{-1}(u(q)(T)-g)$ belongs to $H^{2}(\Omega)$, and thus the regularity of the initial guess $q^{0}$ essentially determines the regularity of the iterates, and the algorithm is most suitable for recovering a smooth potential.

Remark 4.2. There are alternative choices of fixed point algorithms. One popular choice is due to Isakov [13]: given the initial guess $q^{0}$, it reads

$$
q^{k+1}=\frac{\partial_{t}^{\alpha} u\left(q^{k}\right)(T)-\Delta u\left(q^{k}\right)(T)}{g} .
$$

The convergence of the algorithm in the time-fractional case has been analyzed in [42], provided that the terminal time $T$ is sufficiently large. Anderson acceleration might also be used to accelerate this algorithm.

\section{Numerical reconstructions and discussions}

Now we illustrate the accuracy and efficiency of the fixed point algorithm (4.1) with oneand two-dimensional numerical examples. The direct problem is solved by a fully discrete scheme based on the Galerkin finite element method in space and backward Euler convolution quadrature in time, which is first-order accurate in time and second-order accurate in space [15]; (see [16] for an overview of existing schemes). The noisy data $g$ is generated by

$$
g(x)=u\left(q^{\dagger}\right)(x, T)+\epsilon \sup _{x \in \Omega}\left|u\left(q^{\dagger}\right)(x, T)\right| \xi(x), \quad x \in \Omega,
$$

where the noise $\xi(x)$ follows the standard Gaussian distribution, and $\epsilon \geqslant 0$ denotes the (relative) noise level. The exact data $u\left(q^{\dagger}\right)(x, T)$ is generated using a finer spatial-temporal mesh in order to avoid the inverse crime. In Anderson acceleration, the memory parameter $m$ is fixed at 2 , and the relaxation parameter $\lambda$ is fixed at 1000 and 100 for one- and two-dimensional problems, respectively. Note that this choice of $\lambda$ is not optimized, since the optimal choice depends strongly on the problem data, e.g., $T$ and $u_{0}$. Nonetheless, the numerical experiments below indicate that Anderson acceleration is fairly robust with respect to $\lambda$, and works for a broad range of $\lambda$ values. Throughout, the parameter $\tau$ in the discrepancy principle (4.3) is fixed at $\tau=1.01$. Below, For a given reconstruction $q^{*}$, we compute two metrics, the $L^{2}(\Omega)$-error $e_{q}$ and the residual $r_{q}$, defined, respectively, by

$$
e_{q}=\left\|q^{\dagger}-q^{*}\right\|_{L^{2}(\Omega)} \quad \text { and } \quad r_{q}=\left\|u\left(q^{*}\right)-g\right\|_{L^{2}(\Omega)},
$$

where $q^{\dagger}$ denotes the exact potential. Unless otherwise specified, the results presented below are obtained by the fixed point algorithm (4.1) with Anderson acceleration, with a zero initial guess. 
Table 1. The reconstruction error $e_{q}$ for example 5.1 with $T=0.01$.

\begin{tabular}{cccccc}
\hline$\alpha \backslash \epsilon$ & 0 & \multicolumn{1}{c}{$1 \times 10^{-3}$} & \multicolumn{1}{c}{$5 \times 10^{-3}$} & \multicolumn{1}{c}{$1 \times 10^{-2}$} & $5 \times 10^{-2}$ \\
\hline 0.25 & $2.21 \times 10^{-3}(1000)$ & $4.77 \times 10^{-2}(8)$ & $1.36 \times 10^{-1}(7)$ & $2.04 \times 10^{-1}(4)$ & $3.91 \times 10^{-1}(3)$ \\
0.50 & $2.71 \times 10^{-3}(1000)$ & $4.43 \times 10^{-2}(15)$ & $1.41 \times 10^{-1}(7)$ & $1.99 \times 10^{-1}(4)$ & $4.06 \times 10^{-1}(3)$ \\
0.75 & $2.01 \times 10^{-3}(1000)$ & $4.91 \times 10^{-2}(7)$ & $9.06 \times 10^{-2}(6)$ & $2.02 \times 10^{-1}(3)$ & $1.04 \times 10^{0}(1)$ \\
1.00 & $3.36 \times 10^{-3}(1000)$ & $7.85 \times 10^{-2}(6)$ & $2.12 \times 10^{-1}(3)$ & $2.83 \times 10^{-1}(3)$ & $1.00 \times 10^{0}(1)$ \\
\hline
\end{tabular}

Table 2. The reconstruction error $e_{q}$ for example 5.1 with $T=0.1$.

\begin{tabular}{cccccc}
\hline$\alpha \backslash \epsilon$ & 0 & $1 \times 10^{-3}$ & $5 \times 10^{-3}$ & $1 \times 10^{-2}$ & $5 \times 10^{-2}$ \\
\hline 0.25 & $2.89 \times 10^{-3}(1000)$ & $4.69 \times 10^{-2}(9)$ & $8.06 \times 10^{-2}(9)$ & $2.04 \times 10^{-1}(4)$ & $3.88 \times 10^{-1}(3)$ \\
0.50 & $3.65 \times 10^{-3}(1000)$ & $4.96 \times 10^{-2}(8)$ & $8.10 \times 10^{-2}(7)$ & $2.04 \times 10^{-1}(4)$ & $3.78 \times 10^{-1}(3)$ \\
0.75 & $5.44 \times 10^{-3}(1000)$ & $4.69 \times 10^{-2}(9)$ & $7.95 \times 10^{-2}(8)$ & $2.03 \times 10^{-1}(4)$ & $3.50 \times 10^{-1}(3)$ \\
1.00 & $4.99 \times 10^{-3}(1000)$ & $4.59 \times 10^{-2}(9)$ & $7.08 \times 10^{-2}(8)$ & $2.01 \times 10^{-1}(4)$ & $3.19 \times 10^{-1}(3)$ \\
\hline
\end{tabular}

Table 3. The reconstruction error $e_{q}$ for example 5.1 with $T=1$.

\begin{tabular}{lcllll}
\hline$\alpha \backslash \epsilon$ & \multicolumn{1}{c}{$1 \times 10^{-3}$} & \multicolumn{1}{c}{$5 \times 10^{-3}$} & \multicolumn{1}{c}{$1 \times 10^{-2}$} & \multicolumn{1}{c}{$5 \times 10^{-2}$} \\
\hline $0.253 .69 \times 10^{-3}(1000)$ & $4.56 \times 10^{-2}(16)$ & $9.55 \times 10^{-2}(10)$ & $2.03 \times 10^{-1}(4)$ & $3.99 \times 10^{-1}(3)$ \\
$0.505 .14 \times 10^{-3}(1000)$ & $4.74 \times 10^{-2}(11)$ & $1.32 \times 10^{-1}(9)$ & $2.02 \times 10^{-1}(4)$ & $4.10 \times 10^{-1}(3)$ \\
$0.751 .19 \times 10^{-2}(1000)$ & $4.93 \times 10^{-2}(12)$ & $7.85 \times 10^{-2}(10)$ & $1.96 \times 10^{-1}(4)$ & $3.54 \times 10^{-1}(4)$ \\
$1.002 .12 \times 10^{-1}(1000)$ & $2.12 \times 10^{-1}(1000)$ & $2.13 \times 10^{-1}(1000)$ & $2.17 \times 10^{-1}(46)$ & $3.14 \times 10^{-1}(43)$ \\
\hline
\end{tabular}

\subsection{Results for the one-dimensional case}

First we present two one-dimensional examples on the unit interval $\Omega=(0,1)$. In the computation, the domain $\Omega$ is divided into $M$ equal subintervals, and the time interval $(0, T)$ is divided into $N$ subintervals. To generate data, we take $M=1000$ and $N=1000$, whereas for the inversion, $M=200$ and $N=500$. The fixed point iteration (4.1) is run for at most 1000 iterations.

The first example is to recover a smooth potential.

Example 5.1. $u_{0}=\sin \pi x+\frac{1}{100} x(1-x)$ and $q^{\dagger}(x)=\mathrm{e}^{x} \sin (2 \pi x)$.

Note that the initial condition $u_{0}$ is chosen to fulfill the conditions in theorem 1.1. The numerical results for example 5.1 are shown in tables 1-3, with three different final times, $T=0.01, T=0.1$ and $T=1$, which also include the results for normal diffusion (i.e. $\alpha=1.00)$. In the tables, the numbers refer to the reconstruction error $e_{q}$, and the numbers in the brackets denote the stopping index determined by the discrepancy principle (4.3). It is observed that the error $e_{q}$ decreases steadily as the noise level $\epsilon$ tends to zero for all three fractional orders $\alpha$ and final time $T$. For each fixed $T$ and $\epsilon$, the accuracy does not change much with respect to $\alpha$, and thus the fractional order $\alpha$ does not influence much the behavior of the reconstruction error. Nonetheless, for any fixed $\alpha$, when the data is noise free, the error $e_{q}$ increases with the time $T$, although only very slightly. These observations are consistent with the local Lipschitz stability in theorem 1.1 (and the stability for the parabolic case [6]), which holds for all $\alpha \in(0,1]$, so long as the terminal time $T$ is sufficiently small. The numerical experiments actually indicate that even for much large $T$, the inverse problem exhibits nearly identical behavior in terms of the reconstruction error $e_{q}$, indicating similar degree of 


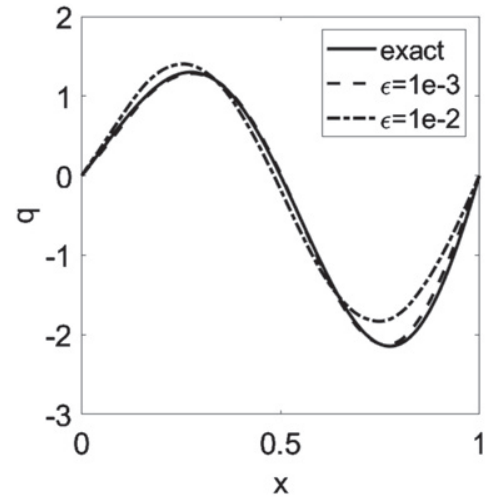

(a) $\alpha=0.25$

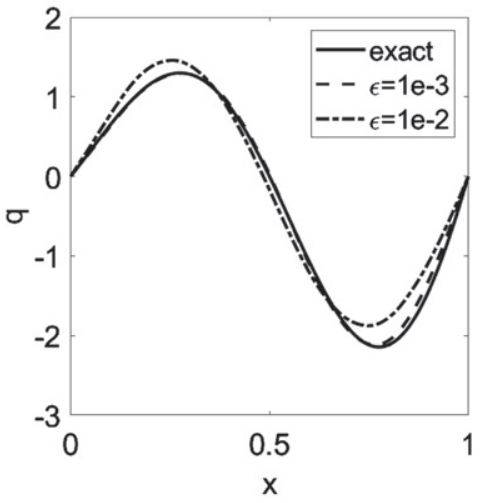

(c) $\alpha=0.75$

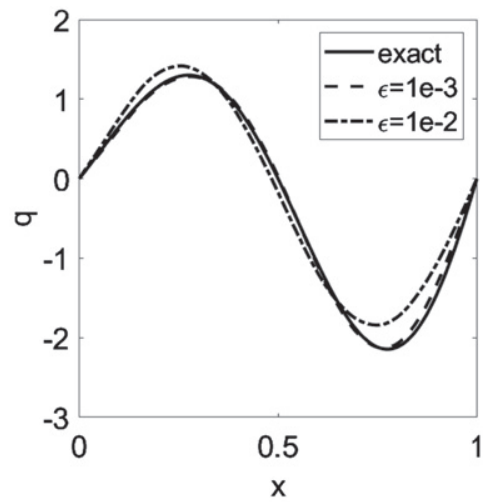

(b) $\alpha=0.50$

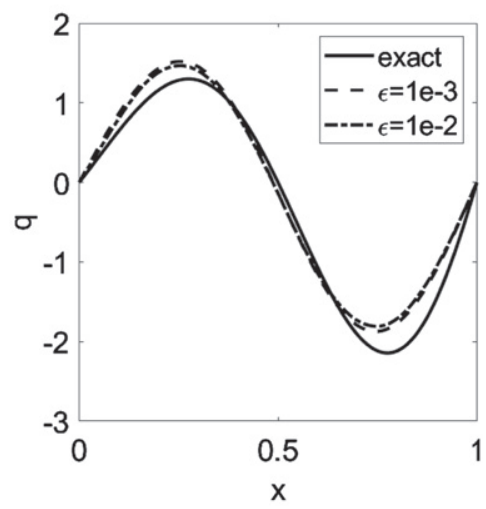

(d) $\alpha=1.00$

Figure 2. Numerical reconstructions for example 5.1 at $T=1$ with different $\alpha$ values.

ill-posedness. See figure 2 for exemplary reconstructions for example 5.1 with $T=1$ at two noise levels. The reconstructions are largely comparable with each other for different fractional orders, corroborating table 3 . However, the last observation for large $T$ seems no longer valid for normal diffusion (i.e., $\alpha=1$ ), for which the numerical reconstruction becomes much more challenging; the fixed point algorithm does not work as well as in the fractional case: it takes many more iterations to reach the discrepancy principle, and yet the reconstruction is generally inferior at all noise levels. This agree also with the empirical observations in the last column of figure 1 of [20].

Tables 1-3 indicate that with Anderson acceleration and discrepancy principle, the fixed point algorithm is generally terminated after about 10 iterations for low noise level, and 5 iterations for high noise levels. In contrast, the fixed point algorithm (4.1) takes far more iterations, by a factor of 10 ; see table 4 for related results for example 5.1 with $T=1$. Nonetheless, with or without acceleration, the obtained reconstruction errors are largely comparable with each other, except the case $\epsilon=0$, for which the iteration (4.1) requires far more than 1000 iterations in order to achieve comparable accuracy with that in table 3. Thus, Anderson acceleration is very effective in speeding up the convergence, while maintaining comparable accuracy. It is worth noting that for $T=1$, the results for normal diffusion are inferior for $T=1$, as manifested by the fact that the convergence of the fixed point algorithm suffers seriously and the least-squares problem in Anderson acceleration exhibits pronounced ill-conditioning, which 
Table 4. The reconstruction error $e_{q}$ for example 5.1 with $T=1$, without Anderson acceleration.

\begin{tabular}{cccccc}
\hline$\alpha \backslash \epsilon$ & 0 & $1 \times 10^{-3}$ & $5 \times 10^{-3}$ & $1 \times 10^{-2}$ & $5 \times 10^{-2}$ \\
\hline 0.25 & $2.49 \times 10^{-2}(1000)$ & $4.93 \times 10^{-2}(368)$ & $1.43 \times 10^{-1}(110)$ & $2.19 \times 10^{-1}(75)$ & $8.23 \times 10^{-1}(18)$ \\
0.50 & $3.03 \times 10^{-2}(1000)$ & $4.96 \times 10^{-2}(496)$ & $1.43 \times 10^{-1}(148)$ & $2.17 \times 10^{-1}(101)$ & $8.10 \times 10^{-1}(25)$ \\
0.75 & $4.64 \times 10^{-2}(1000)$ & $5.02 \times 10^{-2}(896)$ & $1.42 \times 10^{-1}(267)$ & $2.15 \times 10^{-1}(182)$ & $7.97 \times 10^{-1}(46)$ \\
1.00 & $1.18 \times 10^{0}$ & $1.18 \times 10^{0}$ & $1.18 \times 10^{0}$ & $1.19 \times 10^{0}$ & $1.19 \times 10^{0}$ \\
& $(1000)$ & $(1000)$ & $(1000)$ & $(1000)$ & $(1000)$ \\
\hline
\end{tabular}

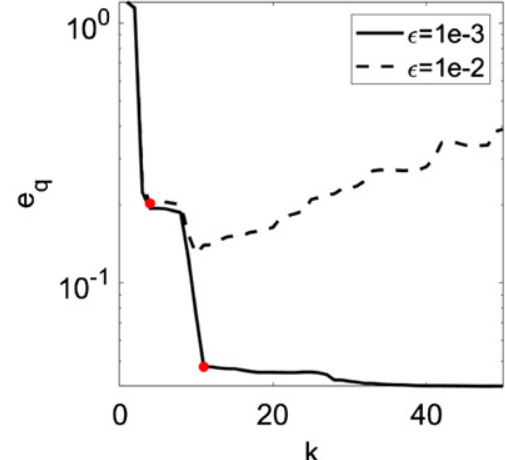

(a) error $e_{q}$

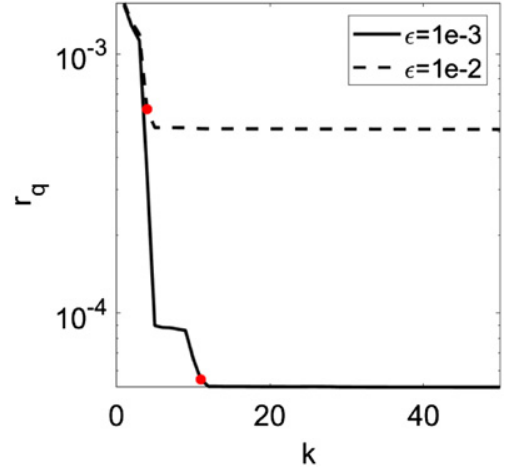

(b) residual $r_{q}$

Figure 3. Convergence behavior of the fixed point algorithm with Anderson acceleration for example 5.1 with $\alpha=0.5$ at $T=1$. In the plots, the red circle refers to the stopping index determined by the discrepancy principle (4.1).

necessitates proper regularization (done via SVD here). Also the accelerating effect of Anderson acceleration is less dramatic, although it does converge after more iterations, when compared with that for smaller $T$ or small $\alpha$. These observations seem to indicate the dramatic difference in the behavior of the inverse potential problem for subdiffusion and normal diffusion at large time $T$, and the fractional case is far more amenable with numerical reconstruction.

The convergence behavior of the acceleration scheme is shown in figure 3 . Note that the reconstruction error $e_{q}$ first decreases, and then starts to increase as the iteration further proceeds. This behavior is very similar to semi-convergence typically observed for an iterative regularization method (e.g., Landweber iteration). The discrepancy principle (4.3) can choose a suitable stopping index before the divergence kicks in, indicated by the red circle in the plots, and the attained reconstruction error is only slightly larger than the optimal value (along the trajectory), showing the optimality of the discrepancy principle. Further, a few extra iterations beyond the stopping index does not greatly deteriorate the reconstruction, i.e., the algorithm enjoys excellent numerical stability. This highly desirable property is attributed to the use of the preconditioner $A^{-1}$ in the iteration (4.1). Surprisingly, the residual $r_{q}$ is monotonically decreasing as the iteration proceeds, and eventually levels off at the noise level $\delta$. That is, the fixed point iteration is actually a descent method for minimizing the residual $r_{q}$, an interesting fact that remains to be rigorously established (see proposition 4.2 for a partial justification). Thus, overall, the algorithm with discrepancy principle is an effective reconstruction method. 
Table 5. The reconstruction error $e_{q}$ for example 5.2 with $T=0.01$.

\begin{tabular}{cccccc}
\hline$\alpha \backslash \epsilon$ & 0 & $1 \times 10^{-3}$ & $5 \times 10^{-3}$ & $1 \times 10^{-2}$ & $5 \times 10^{-2}$ \\
\hline 0.25 & $3.53 \times 10^{-3}(1000)$ & $2.68 \times 10^{-2}(5)$ & $3.70 \times 10^{-2}(2)$ & $3.95 \times 10^{-2}(2)$ & $7.59 \times 10^{-2}(2)$ \\
0.50 & $7.48 \times 10^{-3}(1000)$ & $2.56 \times 10^{-2}(5)$ & $4.02 \times 10^{-2}(2)$ & $4.54 \times 10^{-2}(2)$ & $1.13 \times 10^{-1}(2)$ \\
0.75 & $1.63 \times 10^{-2}(1000)$ & $2.57 \times 10^{-2}(4)$ & $3.81 \times 10^{-2}(2)$ & $4.99 \times 10^{-2}(2)$ & $1.79 \times 10^{-1}(2)$ \\
1.00 & $1.18 \times 10^{-1}(1000)$ & $1.46 \times 10^{-1}(8)$ & $1.35 \times 10^{-1}(1)$ & $1.16 \times 10^{-1}(1)$ & $1.18 \times 10^{-1}(1)$ \\
\hline
\end{tabular}

Table 6. The reconstruction error $e_{q}$ for example 5.2 with $T=0.1$.

\begin{tabular}{cccccc}
\hline$\alpha \backslash \epsilon$ & 0 & $1 \times 10^{-3}$ & $5 \times 10^{-3}$ & $1 \times 10^{-2}$ & $5 \times 10^{-2}$ \\
\hline 0.25 & $3.25 \times 10^{-3}(1000)$ & $1.95 \times 10^{-2}(6)$ & $3.53 \times 10^{-2}(2)$ & $3.69 \times 10^{-2}(2)$ & $1.11 \times 10^{-1}(1)$ \\
0.50 & $7.58 \times 10^{-3}(1000)$ & $2.79 \times 10^{-2}(5)$ & $3.76 \times 10^{-2}(2)$ & $3.96 \times 10^{-2}(2)$ & $6.91 \times 10^{-2}(2)$ \\
0.75 & $9.57 \times 10^{-3}(1000)$ & $2.83 \times 10^{-2}(5)$ & $4.45 \times 10^{-2}(4)$ & $4.55 \times 10^{-2}(2)$ & $7.91 \times 10^{-2}(2)$ \\
1.00 & $1.94 \times 10^{-2}(1000)$ & $1.87 \times 10^{-2}(5)$ & $3.67 \times 10^{-2}(2)$ & $3.85 \times 10^{-2}(2)$ & $6.91 \times 10^{-2}(2)$ \\
\hline
\end{tabular}

Table 7. The reconstruction error $e_{q}$ for example 5.2 with $T=1$.

\begin{tabular}{cccccc}
\hline$\alpha \backslash \epsilon$ & 0 & $1 \times 10^{-3}$ & $5 \times 10^{-3}$ & $1 \times 10^{-2}$ & $5 \times 10^{-2}$ \\
\hline 0.25 & $3.21 \times 10^{-3}(1000)$ & $1.69 \times 10^{-2}(6)$ & $3.43 \times 10^{-2}(2)$ & $3.53 \times 10^{-2}(2)$ & $5.66 \times 10^{-2}(1)$ \\
0.50 & $6.52 \times 10^{-3}(1000)$ & $2.79 \times 10^{-2}(7)$ & $3.44 \times 10^{-2}(2)$ & $3.52 \times 10^{-2}(2)$ & $4.99 \times 10^{-2}(2)$ \\
0.75 & $1.06 \times 10^{-2}(1000)$ & $3.04 \times 10^{-2}(6)$ & $4.36 \times 10^{-2}(5)$ & $3.50 \times 10^{-2}(2)$ & $4.57 \times 10^{-2}(2)$ \\
1.00 & $6.02 \times 10^{-2}(1000)$ & $6.02 \times 10^{-2}(1000)$ & $6.04 \times 10^{-2}(5)$ & $6.06 \times 10^{-2}(5)$ & $6.06 \times 10^{-2}(4)$ \\
\hline
\end{tabular}

The next example is about recovering a nonsmooth coefficient.

Example 5.2. $u_{0}(x)=1+\frac{3}{2} \sin 2 \pi x$ and $q^{\dagger}(x)=\min (x, 1-x)$.

Note that the given initial condition $u_{0}$ does not satisfy the condition of theorem 1.1 , since it does not satisfy the required regularity condition and also changes sign in the domain, and the true potential $q^{\dagger}$ is also less smooth. The numerical results for example 5.2 are summarized in tables 5-7. Similar to example 5.1, it is observed that the fractional order $\alpha$ and the terminal time $T$ does not affect much the reconstruction accuracy, indicating generic ill-posedness of the inverse problem, irrespective of the fractional order $\alpha$. See figure 4 for numerical reconstructions for the case $T=1$; and like before, the results for the case $\alpha=1.00$ are inferior to that in the fractional case. Overall, the reconstructions represent acceptable approximations. Unsurprisingly, the approximation error is largely around the kink, where the exact potential $q^{\dagger}$ exhibits weak singularity. This is attributed to the smoothing effect of the preconditioner $A^{-1}$ in the fixed point update. Thus, the iterates are overly smooth when compared with the exact one $q^{\dagger}$.

According to tables 5-7, the overall algorithm converges within 5 iterations. In contrast, the convergence of the fixed point algorithm (4.1) requires many more iterations; see table 8 for example 5.2 with $T=1$. Nonetheless, except for the case $\alpha=1.00$, the reconstruction errors are largely comparable. Thus, Anderson acceleration is also effective in speeding up the convergence for recovering nonsmooth potentials. The plots of iterate convergence in figure 5 again show a clear semi-convergence phenomenon. Note that overall the fixed point iterates is still descent with respect to the residual, although there is one oscillation at the beginning. The oscillation is related to the fact that the chosen $\lambda$ is fairly large. 


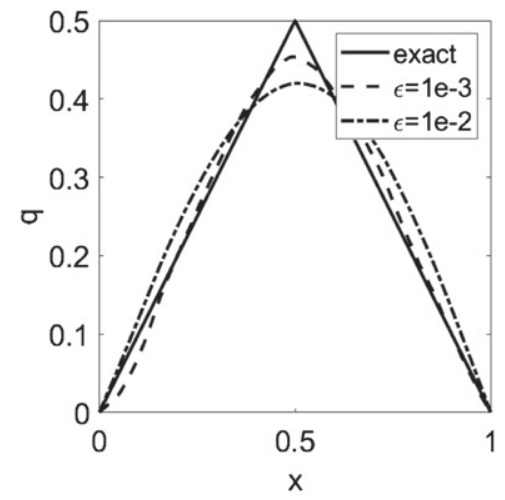

(a) $\alpha=0.25$

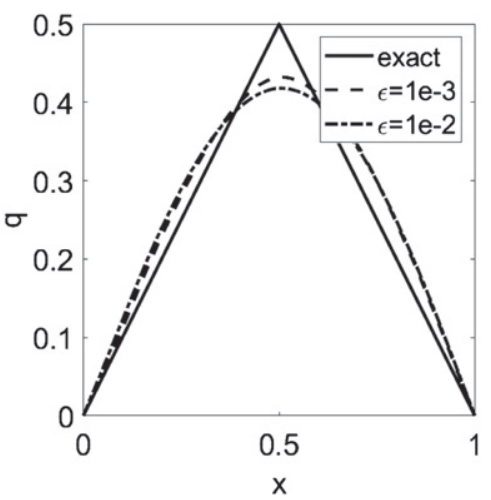

(c) $\alpha=0.75$

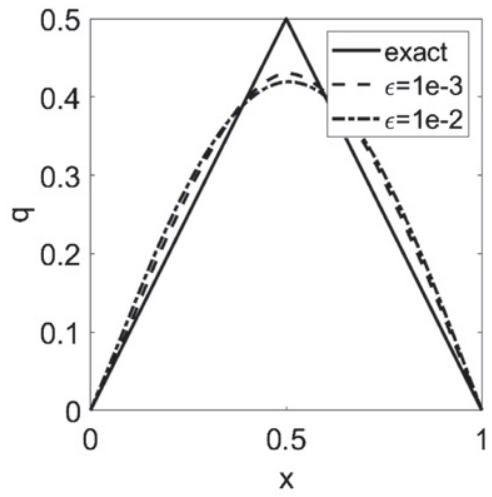

(b) $\alpha=0.50$

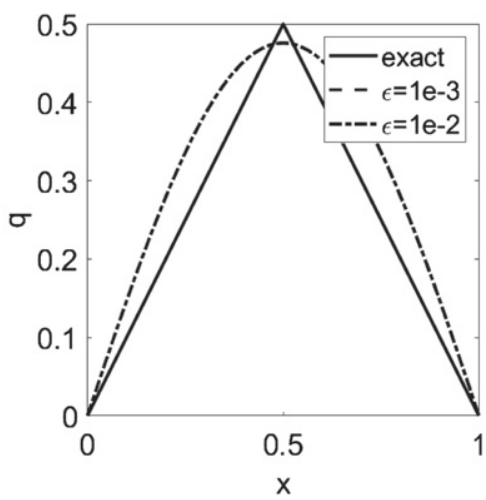

(d) $\alpha=1.00$

Figure 4. Numerical reconstructions for example 5.2 at $T=1$ with different $\alpha$ values.

Table 8. The reconstruction error $e_{q}$ for example 5.2 with $T=1$, without Anderson acceleration.

\begin{tabular}{cccccc}
\hline$\alpha \backslash \epsilon$ & 0 & $1 \times 10^{-3}$ & $5 \times 10^{-3}$ & $1 \times 10^{-2}$ & $5 \times 10^{-2}$ \\
\hline 0.25 & $6.85 \times 10^{-3}(1000)$ & $2.67 \times 10^{-2}(43)$ & $3.39 \times 10^{-2}(3)$ & $3.49 \times 10^{-2}(3)$ & $5.66 \times 10^{-2}(1)$ \\
0.50 & $8.24 \times 10^{-3}(1000)$ & $2.82 \times 10^{-2}(51)$ & $3.43 \times 10^{-2}(5)$ & $3.46 \times 10^{-2}(4)$ & $4.43 \times 10^{-2}(2)$ \\
0.75 & $1.16 \times 10^{-2}(1000)$ & $3.03 \times 10^{-2}(79)$ & $3.45 \times 10^{-2}(9)$ & $3.44 \times 10^{-2}(7)$ & $4.51 \times 10^{-2}(4)$ \\
1.00 & $5.65 \times 10^{-2}$ & $5.66 \times 10^{-2}$ & $5.67 \times 10^{-2}$ & $5.70 \times 10^{-2}$ & $5.61 \times 10^{-2}$ \\
& $(1000)$ & $(1000)$ & $(1000)$ & $(1000)$ & $(864)$ \\
\hline \multicolumn{7}{c}{}
\end{tabular}

\subsection{Results for the two-dimensional case}

Last we given a two-dimensional example on the unit square $\Omega=(0,1)^{2}$ with a smooth coefficient. The domain $\Omega$ is first partitioned smaller square of side length $1 / M$, and then a uniform triangulation is obtained by connecting the upper left and lower right vertices. The data is generated using $M=200$ and $N=1000$, and for the inversion, the discretization parameters are taken to be $M=100$ and $N=500$. The fixed point algorithm (4.1) is run for a maximum 200 iterations and the relaxation parameter $\lambda$ is fixed at 100, which is very conservative for scheme (4.1).

Example 5.3. $u_{0}\left(x_{1}, x_{2}\right)=\sin ^{2} \pi x_{2}$ and $q^{\dagger}\left(x_{1}, x_{2}\right)=\sin \left(\pi x_{1}\right) x_{2}\left(1-x_{2}\right)$. 


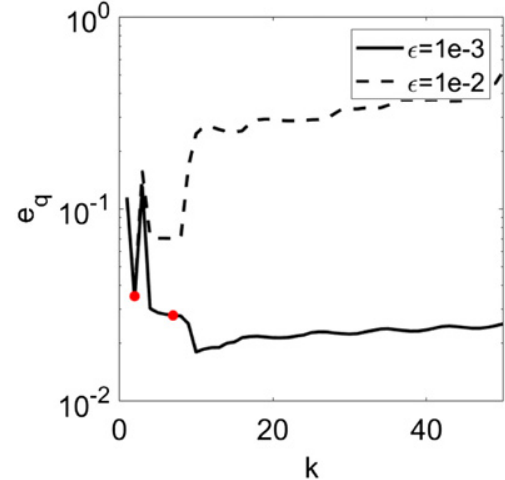

(a) error $e_{q}$

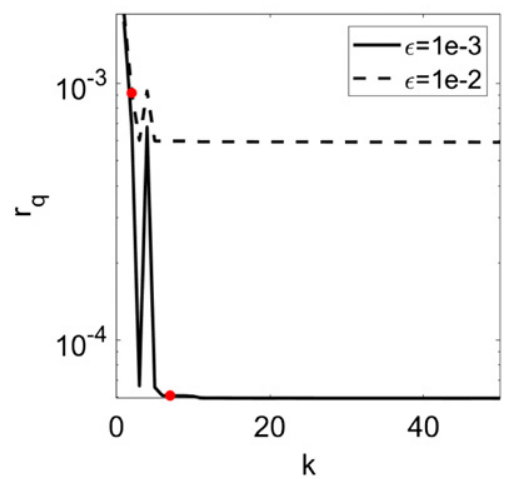

(b) residual $r_{q}$

Figure 5. Convergence behavior of the fixed point algorithm with Anderson acceleration for example 5.2 with $\alpha=0.5$. In the plots, the red circle refers to the stopping index determined by the discrepancy principle (4.1).

Table 9. The reconstruction error $e_{q}$ for example 5.3 at $T=0.1$.

\begin{tabular}{cccccc}
\hline$\alpha \backslash \epsilon$ & 0 & $1 \times 10^{-3}$ & $5 \times 10^{-3}$ & $1 \times 10^{-2}$ & $3 \times 10^{-2}$ \\
\hline 0.25 & $2.29 \times 10^{-3}(200)$ & $6.27 \times 10^{-3}(2)$ & $6.91 \times 10^{-3}(2)$ & $8.16 \times 10^{-3}(2)$ & $1.52 \times 10^{-2}(2)$ \\
0.50 & $5.59 \times 10^{-3}(200)$ & $6.11 \times 10^{-3}(2)$ & $6.23 \times 10^{-3}(2)$ & $6.94 \times 10^{-3}(2)$ & $1.30 \times 10^{-2}(2)$ \\
0.75 & $1.13 \times 10^{-2}(200)$ & $8.03 \times 10^{-3}(2)$ & $7.55 \times 10^{-3}(2)$ & $7.25 \times 10^{-3}(2)$ & $9.48 \times 10^{-3}(2)$ \\
1.00 & $1.37 \times 10^{-2}(200)$ & $1.19 \times 10^{-2}(5)$ & $1.03 \times 10^{-2}(2)$ & $9.83 \times 10^{-3}(2)$ & $8.95 \times 10^{-3}(2)$ \\
\hline
\end{tabular}
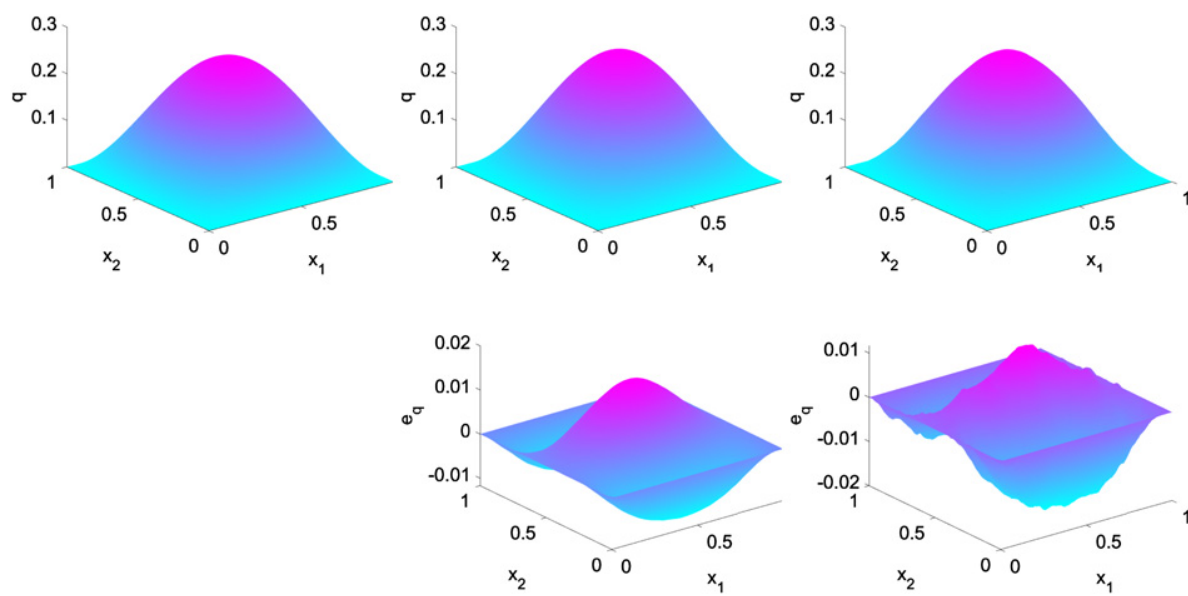

(a) exact $q^{\dagger}$

(b) $\epsilon=1 \mathrm{e}-3$

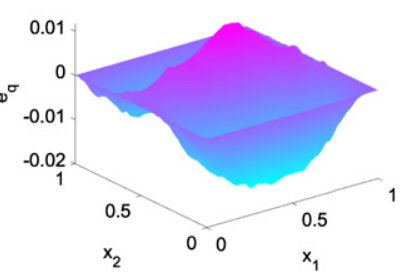

(c) $\epsilon=1 \mathrm{e}-2$

Figure 6. Numerical results for example 5.3 with $T=0.1$ and $\alpha=0.5$ : the first and second rows refer to the reconstruction $q$ and the pointwise error $e_{q}=q-q^{\dagger}$. 
The initial condition $u_{0}$ does not satisfy the condition in theorem 1.1. The numerical results for example 5.3 at $T=0.1$ are shown in table 9 and figure 6 . With $\lambda=100$, the fixed point method (4.1) can only converge very slowly, and requires thousands of iterations to yield reasonable reconstruction, and thus the corresponding results are not shown. Anderson acceleration can greatly speed up the convergence, so that with any fixed noise level $\epsilon>0$, it converges in two iterations. The method converges steadily, and the reconstruction error $e_{q}$ decreases steadily as the noise level $\epsilon$ tends to zero. Up to $\epsilon=1 \times 10^{-2}$ noise in the data, the result represents an excellent reconstruction of the true potential $q^{\dagger}$.

\section{Conclusion}

In this work, we have presented a study on the inverse problem of recovering a potential in the subdiffusion model from terminal data. Under certain restrictions on the initial data, we have derived a local Lipschitz stability result, using refined properties of Mittag-Leffler functions. Further, we have developed a simple fixed point algorithm for recovering the potential coefficient. When equipped with the discrepancy principle and Anderson acceleration, extensive numerical experiments indicate that it is highly efficient and accurate.

There are a few interesting questions on the inverse potential problem awaiting answers. First, the numerical experiments indicate a descent property of the fixed point iteration for the residual, which however remains to be established in the general case. Second, it is of much interest to analyze the regularizing property, e.g., convergence and convergence rates, of the fixed point algorithm (and the accelerated variant) when equipped with the discrepancy principle. Third, it is natural to ask whether it is possible to recover the potential and the fractional order $\alpha$ simultaneously from the terminal data, and if so, also to derive relevant stability estimates. In the case of lateral Cauchy data, it is known that one can recover the diffusion coefficient and fractional order together [4]. We shall explore these issues in future works.

\section{Acknowledgments}

The authors are grateful to the anonymous referees and the board member for several constructive comments, which have led to an improvement in the quality of the paper.

\section{ORCID iDs}

Bangti Jin (ㄴ) https://orcid.org/0000-0002-3775-9155

Zhi Zhou (1) https://orcid.org/0000-0002-3439-0313

\section{References}

[1] Adams R A and Fournier J J F 2003 Sobolev Spaces 2nd edn (Amsterdam: Elsevier/Academic)

[2] Anderson D G 1965 Iterative procedures for nonlinear integral equations J. ACM 12 547-60

[3] Brezinski C, Redivo-Zaglia M and Saad Y 2018 Shanks sequence transformations and Anderson acceleration SIAM Rev. 60 646-69

[4] Cheng J, Nakagawa J, Yamamoto M and Yamazaki T 2009 Uniqueness in an inverse problem for a one-dimensional fractional diffusion equation Inverse Problems 25115002

[5] Choulli M and Yamamoto M 1996 Generic well-posedness of an inverse parabolic problem - the Hölder-space approach Inverse Problems 12 195-205

[6] Choulli M and Yamamoto M 1997 An inverse parabolic problem with non-zero initial condition Inverse Problems 13 19-27 
[7] Ciarlet P G 2013 Linear and Nonlinear Functional Analysis with Applications (Philadelphia, PA: SIAM)

[8] Courant R and Hilbert D 1953 Methods of Mathematical Physics vol 1 (New York: Interscience)

[9] Engl H W, Hanke M and Neubauer A 1996 Regularization of Inverse Problems (Dordrecht: Kluwer)

[10] Evans C, Pollock S, Rebholz L G and Xiao M 2020 A proof that Anderson acceleration improves the convergence rate in linearly converging fixed-point methods (but not in those converging quadratically) SIAM J. Numer. Anal. 58 788-810

[11] Hatano Y and Hatano N 1998 Dispersive transport of ions in column experiments: an explanation of long-tailed profiles Water Resour. Res. 34 1027-33

[12] Henry B I, Langlands T A M and Wearne S L 2006 Anomalous diffusion with linear reaction dynamics: from continuous time random walks to fractional reaction-diffusion equations Phys. Rev. E 74031116

[13] Isakov V 1991 Inverse parabolic problems with the final overdetermination Commun. Pure Appl. Math. 44 185-209

[14] Ito K and Jin B 2015 Inverse Problems: Tikhonov Theory and Algorithms (Singapore: World Scientific)

[15] Jin B, Lazarov R and Zhou Z 2016 Two fully discrete schemes for fractional diffusion and diffusionwave equations with nonsmooth data SIAM J. Sci. Comput. 38 A146-70

[16] Jin B, Lazarov R and Zhou Z 2019 Numerical methods for time-fractional evolution equations with nonsmooth data: a concise overview Comput. Methods Appl. Mech. Eng. 346 332-58

[17] Jin B, Li B and Zhou Z 2018 Numerical analysis of nonlinear subdiffusion equations SIAM J. Numer. Anal. 56 1-23

[18] Jin B and Rundell W 2012 An inverse problem for a one-dimensional time-fractional diffusion problem Inverse Problems 28075010

[19] Jin B and Rundell W 2015 A tutorial on inverse problems for anomalous diffusion processes Inverse Problems 31035003

[20] Kaltenbacher B and Rundell W 2019 On an inverse potential problem for a fractional reactiondiffusion equation Inverse Problems 35065004

[21] Kian Y and Yamamoto M 2019 Reconstruction and stable recovery of source terms and coefficients appearing in diffusion equations Inverse Problems $\mathbf{3 5} 115006$

[22] Kilbas A A, Srivastava H M and Trujillo J J 2006 Theory and Applications of Fractional Differential Equations (North-Holland Mathematics Studies vol 204) (Amsterdam: Elsevier)

[23] Klibanov M V, Li J and Zhang W 2020 Convexification for an inverse parabolic problem arXiv:2001.01880

[24] Krasnoschok M V 2016 Solvability in holder space of an initial boundary value problem for the time-fractional diffusion Z. Mat. Fiz. Anal. Geom. 12 48-77

[25] Li Z, Cheng X and Liu Y 2020 Generic well-posedness for an inverse source problem for a multiterm time-fractional diffusion equation Taiwan. J. Math. 24 1005-20

[26] Liu Y, Li Z and Yamamoto M 2019 Inverse problems of determining sources of the fractional partial differential equations Handbook of Fractional Calculus with Applications vol 2 (Berlin: de Gruyter \& Co) pp 411-29

[27] Liu Y, Rundell W and Yamamoto M 2016 Strong maximum principle for fractional diffusion equations and an application to an inverse source problem Fract. Calc. Appl. Anal. 19 888-906

[28] Luchko Y and Yamamoto M 2017 On the maximum principle for a time-fractional diffusion equation Fract. Calc. Appl. Anal. 20 1131-45

[29] Metzler R and Klafter J 2000 The random walk's guide to anomalous diffusion: a fractional dynamics approach Phys. Rep. 33977

[30] Miller L and Yamamoto M 2013 Coefficient inverse problem for a fractional diffusion equation Inverse Problems 29075013

[31] Nigmatulin R R 1986 The realization of the generalized transfer equation in a medium with fractal geometry Phys. Status Solidi B 133 425-30

[32] Pollard H 1948 The completely monotonic character of the Mittag-Leffler function $E_{\mathrm{a}}(-x) B u l l$. Am. Math. Soc. 54 1115-7

[33] Prilepko A I and Solov'ev V V 1987 On the solvability of inverse boundary value problems for the determination of the coefficient preceding the lower derivative in a parabolic equation Differentsial' nye Uravneniya 23 136-43

[34] Rundell W 1987 The determination of a parabolic equation from initial and final data Proc. Am. Math. Soc. 99637 
[35] Sakamoto K and Yamamoto M 2011 Initial value/boundary value problems for fractional diffusionwave equations and applications to some inverse problems J. Math. Anal. Appl. 382 426-47

[36] Sakamoto K, Yamamoto M and Yamamoto M 2011 Inverse source problem with a final overdetermination for a fractional diffusion equation Math. Contr. Relat. Field. 1 509-18

[37] Seybold H and Hilfer R 2008/09 Numerical algorithm for calculating the generalized Mittag-Leffler function SIAM J. Numer. Anal. 47 69-88

[38] Simon T 2014 Comparing Fréchet and positive stable laws Electron. J. Probab. 1925

[39] Toth A and Kelley C T 2015 Convergence analysis for Anderson acceleration SIAM J. Numer. Anal. $53805-19$

[40] Walker H F and Ni P 2011 Anderson acceleration for fixed-point iterations SIAM J. Numer. Anal. 49 1715-35

[41] Yuste S B, Abad E and Lindenberg K 2010 Reaction-subdiffusion model of morphogen gradient formation Phys. Rev. E 82061123

[42] Zhang Z and Zhou Z 2017 Recovering the potential term in a fractional diffusion equation IMA J. Appl. Math. 82 579-600 